\author{
功能性可拉伸有机电子器件的研究进展 \\ 边洋爽 ${ }^{a, b}$ 刘凯 ${ }^{a, b}$ 郭云龙 $*, a, b$ 刘云圻 ${ }^{a, b}$ \\ ( ${ }^{a}$ 中国科学院化学研究所有机固体重点实验室 北京 100190) \\ $\left({ }^{b}\right.$ 中国科学院大学 化学科学学院 北京 100049)
}

\begin{abstract}
摘要 可拉伸有机电子器件具有高机械稳定性、优异的电学稳定性、低成本和生物兼容性好等优点, 是未来电子器件 发展的重要方向. 功能性可拉伸有机电子器件更是为可穿戴和可植入设备、智能医疗以及软体机器人等新兴高技术领 域提供了新的研究思路. 本文综述了近年来功能性可拉伸有机电子器件的研究进展, 包括场效应、光电、存储以及传 感等有机晶体管, 发光二极管、交流电致发光、发光电化学电池等有机光电器件, 太阳能电池、超级电容器、纳米发 电机等有机能源存储与转换器件, 压力、应变、触觉、温度、气体等有机传感器, 忆阻器、磁存储、仿突触存储等有 机存储器, 以及其他集成电路系统元件, 最后就功能性可拉伸有机电子器件存在的科学问题与未来的发展方向提出了 建议.
\end{abstract}

关键词＼cjkstart可拉伸有机电子；有机半导体；有机功能器件；稳定性

\title{
Research Progress in Functional Stretchable Organic Electronic Devices
}

$$
\text { Bian, Yangshuang }{ }^{a, b} \text { Liu, Kai }{ }^{a, b} \text { Guo, Yunlong*,a,b Liu, Yunqi }{ }^{a, b}
$$

( ${ }^{a}$ Key Laboratory of Organic Solid, Institute of Chemistry, Chinese Academy of Sciences, Beijing 100190, China)

( ${ }^{b}$ School of Chemical Sciences, University of Chinese Academy of Sciences, Beijing 100049, China)

\begin{abstract}
Stretchable organic electronic devices are characterized with high mechanical stability, superior electronic stability, low cost, satisfactory biocompatibility, etc., thus having been regarded as an inevitable trend in the development of future electronics. Furthermore, the functional stretchable organic electronic devices provide pathways toward the emerging high-tech fields such as wearable and implantable devices, intelligent medical diagnosis system, software robots, etc. This review focuses on the research advances in functional stretchable organic electronic devices, including stretchable organic transistors (field-effect transistors, phototransistors, memory transistors and sensors), stretchable organic optoelectronic devices (light-emitting diodes, alternating current electroluminescent devices and light-emitting electrochemical cells), stretchable organic energy storage and conversion devices (solar cells, supercapacitors and nanogenerators), stretchable organic sensors (pressure sensors, strain sensors, tactile sensors, temperature sensors, gas sensors and other sensors), stretchable organic memory (resistive memory, magnetic memory and bionic synaptic memory) and other functional stretchable organic electronic devices. Finally, through the analyses of the existing scientific problems and future development of the functional stretchable organic electronic devices, we put forward some suggestions.
\end{abstract}

Keywords stretchable organic electronics; organic semiconductors; organic functional devices; stability

\section{1 引言}

可拉伸电子器件作为一种新兴技术打破了传统平 面刚性电路的限制，进一步扩展了电子学的应用领域, 有望解锁成为下一代可穿戴和可植入电子器件 ${ }^{[1,2]}$. 可 拉伸性是可拉伸电子器件的关键性质, 特别是在与移动 相关的研究中, 需要器件在高机械变形情况下保持相对 稳定的电学性能. 目前实现可拉伸的途径主要包括以下 三种: 传统的几何工程学设计 ${ }^{[3,4]}$ 、将材料嵌入弹性体 内 $^{[5,6]}$ 以及使用本征可拉伸材料 ${ }^{[7,8]}$. 相比传统无机器件 的脆性和结构设计的复杂性, 有机电子器件具有柔性、
易加工、低成本及轻便等突出特点, 成为当今可拉伸电 子学的主流研究方向之一 ${ }^{[9]}$. 此外, 可拉伸有机电子器 件优异的生物相容性使其在电子皮肤、医疗监测、人机 界面等领域展现出广阔的应用前景.

自可拉伸电子学提出以来, 有机电子器件凭借其优 异的机械性能、电学性能和加工性能, 在大面积功能性 电子应用上取得了显著进步. 然而，目前在实现可拉伸 电子器件的高机械应变、高密度、功能整合等方面, 仍 然存在着很大的挑战 ${ }^{[10]}$. 据此, 本文综述了近年来功能 性可拉伸有机电子器件的研究进展, 包括可拉伸有机场 效应晶体管(STOTs)、可拉伸有机光电器件(STOPDs)、

* E-mail: guoyunlong@iccas.ac.cn

Received May 31, 2020; published July 8, 2020.

Project supported by the National Natural Science Foundation of China (Nos. 21922511, 51873216, 61890943, 91833306) and the National Key Research and Development Project (No. 2018YFA0703202).

项目受国家自然科学基金(Nos. 21922511, 51873216, 61890943, 91833306)和国家重点研发计划(No. 2018YFA0703202)资助. 
可拉伸有机能源存储和转换器件(STOEDs)、可拉伸有机 传感器(STOSs) 及可拉伸有机存储器(STOM)等功能性 器件的应用(如图 1).

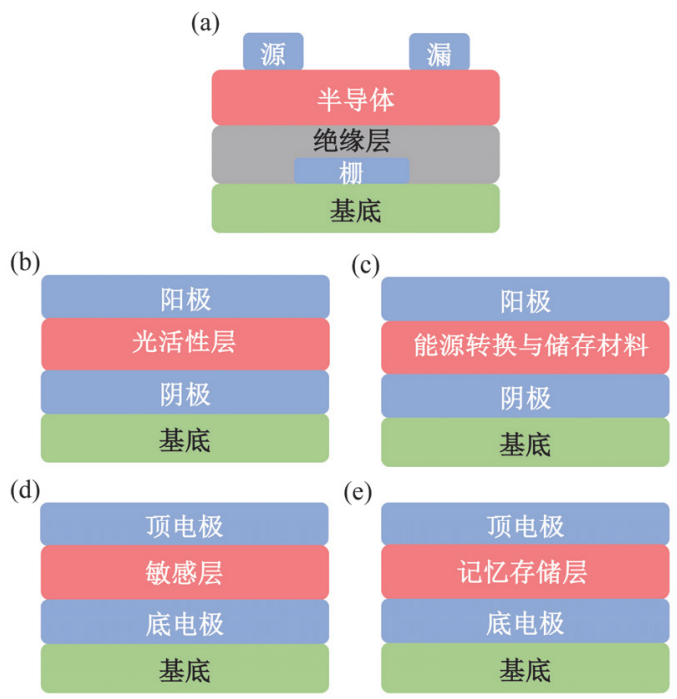

图 1 功能性可拉伸有机电子器件结构示意图

Figure 1 Schematic diagram of the common architectures of functional stretchable organic electronic devices

The common structures of STOTs (a), STOPDs (b), STOEDs (c), STOSs (d) and STOM (e).

\section{2 可拉伸有机晶体管(STOTs)}

作为可变形电子电路的基本元器件, 可拉伸有机晶 体管在传感器寻址、信号读出和放大等方面发挥着重要 作用 ${ }^{[11]}$. 近年来, 有机半导体的机械性能和电学性能都 有了显著提升. 同时, STOTs 也引入了许多新功能以面 对苛刻的实际应用, 包括具有光电特性、存储、传感等 功能性 STOTs, 从而进一步发挥了其内在应用潜力.

\section{1 场效应晶体管}

场效应晶体管器件因具有高阻抗、低噪音、低功耗、 易整合等特点 ${ }^{[12]}$, 受到人们的广泛关注. 可拉伸有机场 效应晶体管(STOFETs)的机械性能及电学性能一直在稳 步提高, 成为非常具发展潜力的可穿戴电子器件.

早前 STOFETs 的研究中, 使用形成微裂纹或孔隙 以适应形变的策略虽然保持了渗透路径 ${ }^{[13]}$, 但也在一 定程度限制了器件的电学性能. Bao 等 ${ }^{[14]}$ 提出了一种基 于共轭聚合物与弹性体相分离(CONPHINE) 产生纳米限 域效应的概念(如图 2a), 即当将聚合物限制在纳米尺度 时, 可以抑制大尺寸晶体的生长, 同时增强无定形区的 链运动. 该方法显著降低了共轭聚合物的模量, 并延迟 了应变裂纹的形成, 因而可以在不影响电荷迁移率的前 提下大幅提高半导体的可拉伸性. 通过该 CONPHINE 薄膜所制备的 STOFETs, 其迁移率可与非晶硅婫美(平 均约 $\left.1.08 \mathrm{~cm}^{2} /(\mathrm{V} \cdot \mathrm{s})\right)$, 即使拉伸到 $100 \%$ 应变下也不受影 响. 此外, Bao 等 ${ }^{[15]}$ 报道了一种溶液剪切法通过结合纳 米限域效应可以实现可拉伸共轭聚合物的多尺度有序
排列，从而大大提高了载流子迁移率。结果表明，该 CONPHINE 薄膜的迁移率提高了三倍，在 $100 \%$ 应变下 也可达到 $1.50 \mathrm{~cm}^{2} /(\mathrm{V} \cdot \mathrm{s})$. 同时，他们将这种多尺度形貌 控制的方法扩展到卷对卷 $(R 2 R) 工$ 艺，真正实现了米量 级高性能、可拉伸半导体薄膜的制备.

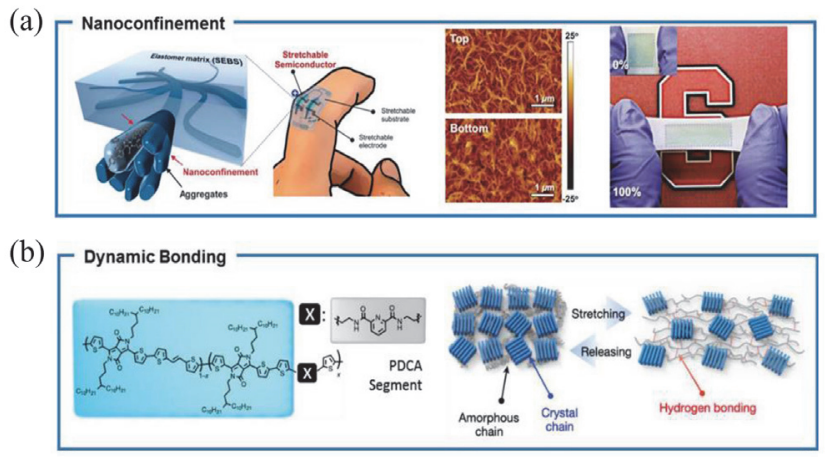

图 2 可拉伸半导体聚合物薄膜

Figure 2 The stretchable semiconducting polymers films (a) Illustration of the nanoconfined effect of semiconducting polymers in an elastomer. (b) Schematic diagram of the stretching and releasing processes of semiconductors films ${ }^{[7]}$. Reprinted with permission from Ref. 7 Copyright (2019) WILEY-VCH.

可拉伸器件面临着持久性差、寿命短和易受机械损 伤等缺点, 从而使器件的性能大打折扣. 因此开发具有 自修复能力的 STOFETs 是目前可拉伸器件的研究热点 之一. Haick 等 ${ }^{[16]}$ 基于碳纳米管 $(\mathrm{CNTs})$ 制备的 STOFETs 实现了高的空穴迁移率 $10 \mathrm{~cm}^{2} /(\mathrm{V} \bullet \mathrm{s})$ 、相对较低的操作 电压 $(<8 \mathrm{~V})$ 和自愈合能力. 该器件将 CNTs 涂覆在含有 动态氢键和二硫键的自愈材料聚氨酯(PUU)中, 通过聚 合物动态链与 CNTs 的相互作用来实现导电通路的再 生. 因此, 无论是在微尺度损伤或器件完全被切断后, 该器件都可以在不借助外部条件下实现自愈合, 并恢复 其电学性能和机械性能. 此外, 在高达 $50 \%$ 的应变下该 器件重复拉伸 $>200$ 个循环后也不会显著降低电学性 能.

本征可拉伸电子器件是实现高机械稳定性、可伸缩 制造和高器件密度的关键. Bao 等 ${ }^{[8]}$ 通过引入化学组分 来促进共轭聚合物的动态非共价交联, 从而实现了一种 本征可拉伸半导体的设计. 基于这种吡咯并吡咯二酮 (DPP)基聚合物的 STOFETs 迁移率高达 $1.3 \mathrm{~cm}^{2} /(\mathrm{V} \cdot \mathrm{s})$, 开关电流比超过 $10^{6}$. 即使在 $100 \%$ 应变下的 100 个循环 测试中, 其迁移率仍然高达 $1.12 \mathrm{~cm}^{2} /(\mathrm{V} \cdot \mathrm{s})$, 这是由于这 种非共价键交联的聚合物分子在应变下可以通过非共 价键的断裂来进行能量耗散(如图 $2 b$ ), 因而可以保持较 高的电荷输运能力. 同时, 该类聚合物通过溶剂和热处 理后还可以实现完全愈合. 表 1 总结了近年来 STOFETs 领域报道的本征可拉伸半导体的设计策略, 包括形成微 裂纹 ${ }^{[13]}$ 、构建动态键 $(\text { 氢键 })^{[8]}$ 、侧链工程 ${ }^{[17,18]}$ 、使用软

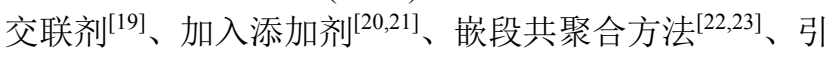
入共轭-断裂片段 ${ }^{[24,25]}$ 以及形成网络状形貌 ${ }^{[26]}$ 等. 
表 1 本征可拉伸有机半导体的设计策略、可拉伸性和器件应用

Table 1 The design strategies, stretchablility and application of intrinsically stretchable organic semiconductors

\begin{tabular}{|c|c|c|c|c|}
\hline Strategies & Active materials & Stretchability $^{a}$ & Application & Ref. \\
\hline Microcracked & P3HT & $160 \%\left(3.4 \times 10^{-2} \mathrm{~cm}^{2} /(\mathrm{V} \cdot \mathrm{s})\right)$ & OFETs & Hwang et al. ${ }^{[13]}$ \\
\hline \multirow[t]{2}{*}{ Nanowire networks } & SWCNT & $50 \%\left(16.2 \mathrm{~cm}^{2} /(\mathrm{V} \cdot \mathrm{s})\right)$ & OFETs & Pei et al. ${ }^{[27]}$ \\
\hline & Sorted semiconducting CNT & $100 \%$ & OFETs & Bao et al. ${ }^{[28]}$ \\
\hline Dynamic Bonding & DPP-based polymer & $100 \%\left(1.12 \times 10^{-2} \mathrm{~cm}^{2} /(\mathrm{V} \cdot \mathrm{s})\right)$ & OFETs & Bao et al. ${ }^{[8]}$ \\
\hline Side-chain Engineering & PTDPPTFT & $100 \%\left(0.1 \mathrm{~cm}^{2} /(\mathrm{V} \cdot \mathrm{s})\right)$ & OFETs & Bao et al. ${ }^{[17]}$ \\
\hline Soft Crosslinkers & DPPTTEC & $100 \%\left(0.4 \mathrm{~cm}^{2} /(\mathrm{V} \cdot \mathrm{s})\right)$ & OFETs & Bao et al. ${ }^{[19]}$ \\
\hline \multirow[t]{2}{*}{ Additives } & CPP/DPP-TT & $100 \%\left(0.14 \mathrm{~cm}^{2} /(\mathrm{V} \cdot \mathrm{s})\right)$ & OFETs & Bao et al. ${ }^{[20]}$ \\
\hline & m-CNT doped P3HTs-NFs/PDMS & $50 \%\left(3.57 \pm 1.30 \mathrm{~cm}^{2} /(\mathrm{V} \cdot \mathrm{s})\right)$ & OFETs & Yu et al. ${ }^{[21]}$ \\
\hline \multirow[t]{2}{*}{ Block Copolymer } & P3HT-PE & $600 \%\left(2 \times 10^{-2} \mathrm{~cm}^{2} /(\mathrm{V} \cdot \mathrm{s})\right)$ & OFETs & Stutzmann et al. ${ }^{[22]}$ \\
\hline & P3HT-PMA-P3HT & $140 \%\left(9 \times 10^{-4} \mathrm{~cm}^{2} /(\mathrm{V} \cdot \mathrm{s})\right)$ & OFETs & Qiu et al. ${ }^{[23]}$ \\
\hline \multirow[t]{2}{*}{ Conjugation-break Spacers } & DPP-based polymer & $100 \%\left(>0.36 \mathrm{~cm}^{2} /(\mathrm{V} \cdot \mathrm{s})\right)$ & OFETs & Bao et al. ${ }^{[24]}$ \\
\hline & DPP-based polymer & $180 \%$ & OFETs & Mei et al. ${ }^{[25]}$ \\
\hline Small grain domains & PTB7:PC71BM:DIO & $100 \%(2.14 \pm 0.63 \%)$ & OSCs & Pei et al. ${ }^{[29]}$ \\
\hline Side-chain Engineering & P3DDT:PCBM & $10 \%(0.381 \pm 0.029 \%)$ & OSCs & Lipomi et al. ${ }^{[30]}$ \\
\hline \multirow[t]{2}{*}{ Crosslinking network } & OF-B/PEO-DMA/LiTf & $45 \%(0.87 \mathrm{~cd} / \mathrm{A})$ & OLEC & Pei et al. ${ }^{[31]}$ \\
\hline & SuperYellow:ETPTA:PEO:LiTf & $120 \%(2.1 \mathrm{~cd} / \mathrm{A})$ & OLEC & Pei et $a l^{[32]}$ \\
\hline Interpenetrating networks & WLEP:OXD-7 & $130 \%$ & OLED & Pei et al. ${ }^{[26]}$ \\
\hline
\end{tabular}

a 括号内表示器件在相应拉伸下的性能值(迁移率/光电转换效率/发光效率).

尽管目前本征可拉伸半导体的开发取得了一定的 进展，但是开发具有高机械延展性和高载流子迁移率的 本征可拉伸半导体仍是未来可拉伸电子器件研究的关 键.

\section{2 光晶体管}

实现开关特性和光电功能的整合是当前功能性 STOTs 研究的热点. 不同于光电二极管, 光晶体管具有 低工作电压、高增益、宽动态范围等特点, 为光电器件 开辟了新的领域. 可拉伸有机光晶体管的柔性、高灵敏 度以及易于整合等特性, 使其在光探测、仿生光学等方 面有很好的应用潜力。

在传统的光电器件中, 单一极性半导体材料中光生 激子仅通过电场分离为自由载流子, 而长的沟道长度 (数十微米)又易引起光生载流子复合 ${ }^{[33]}$, 从而导致光生 载流子分离效率较低. 为此, Guo 等 ${ }^{[34]}$ 基于聚合物体异 质结(PDVT-8/PC 61 BM BHJ)成功地制备了一种新型超短 沟道光晶体管(如图 $3 \mathrm{a}$ ). 他们通过将 $\mathrm{p}$ 型和 $\mathrm{n}$ 型复合材 料作为 D-A 异质结沟道层, 使得本体异质结(BHJ)共混 物中的内建电场有效地增强了光载流子的分离, 因此该 器件对可见光表现出超高灵敏度. 该器件具有良好的光 电性能, 实现了高响应 $718 \mathrm{~A} / \mathrm{W}$, 高光敏性 $4.5 \times 10^{5}$ 以 及高探测能力 $4.1 \times 10^{15}$ Jones $\left(\mathrm{cm} \cdot \mathrm{Hz}^{1 / 2} \cdot \mathrm{W}^{-1}\right)$. 更重要 的是, 在弯曲半径变化 $(r=54 、 27$ 和 $18 \mathrm{~mm})$ 和 1000 次 循环测试中器件的光响应性能仅略有下降(如图 3b, 3c), 这说明该器件具有良好的机械稳定性.

使用量子点来提高器件的光电特性是发展高性能 可拉伸光晶体管的有效途径. Guo 等 ${ }^{[35]}$ 使用钙钛矿量子 点制备了一种具有存储功能的柔性有机光晶体管, 实现 了光编程存储器件. 该器件使用超短通道长度和垂直电 荷转移的结构有效地抑制了捕获载流子效应和界面效 应两个关键影响因素. 基于该器件研制的成像和存储阵 列, 在紫外光编程(紫外光强 $0.50 \mathrm{~mW} / \mathrm{cm}^{2}$ 下保持 $0.5 \mathrm{~s}$ )
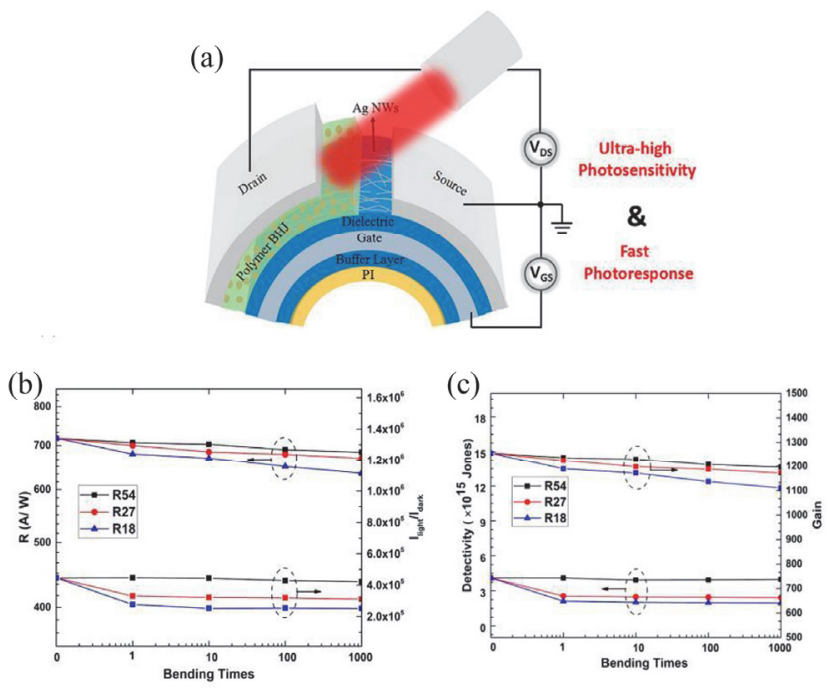

图 3 可拉伸有机光晶体管

Figure 3 The stretchable organic phototransistors (OPTs)

(a) Schematic device structure of PDVT-8/PC61BM OPTs. (b, c) Under different bending times and radius $(r=54,27$, and $18 \mathrm{~mm})$, the variation of responsivity and $I_{\text {light }} / I_{\text {dark }}$ ratio (b) and detectivity and gain (c) ${ }^{[34]}$. Reprinted with permission from Ref. 34 Copyright (2018) American Chemical Society.

和电擦除(源漏电压 $V_{\mathrm{GS}}=-40 \mathrm{~V}$ 下保持 $1 \mathrm{~s}$ ) 的条件下, 实现了 $42 \mathrm{~V}$ 的大存储窗口, 最长保留时间为 $2 \times 10^{5} \mathrm{~s}$. 此外, 由于垂直电荷传输的结构消除了弯曲过程中形成 的裂纹和位错的影响, 该器件具有良好的机械稳定性, 在弯曲半径为 $50 \mathrm{~mm}$ 下, 500 次循环测试中基本保持原 有的电学性能.

\section{3 记忆存储晶体管}

随着信息时代的高速发展, 许多电子器件都需要兼 具存储功能. 有机记忆存储器具有非易失性、可重复读 写、低成本、高容量和轻便等优点, 得到广泛研究. 记 忆存储性 STOTs 也将成为下一代可穿戴电子设备中功 能性器件应用的首选. 
在各种存储器结构中, 铁栅型场效应晶体管具有高 耐久性、非易失性存储和低功耗, 被认为是构造可拉伸 存储器的首选元件. Kim 等 ${ }^{[36]}$ 使用并五苯和 P(VDF-TrFE)构建了一种纤维状可拉伸记忆存储晶体管 (如图 4a). 该器件具有低压运行 $(<5 \mathrm{~V})$ 、开关稳定 $($ 约 100 个周期)以及良好的机械稳定性和准永久保持时间. 当缝在可拉伸的聚丙烯(PP)织物上时, 即使在苛刻的情 况下如单轴(和对角)应变从 0 到 $73.3 \%(100 \%)$ 和随机皱 褶中, 也能实现可靠的数据存储(如图 $4 \mathrm{~b}, 4 \mathrm{c}$ ).

(a)
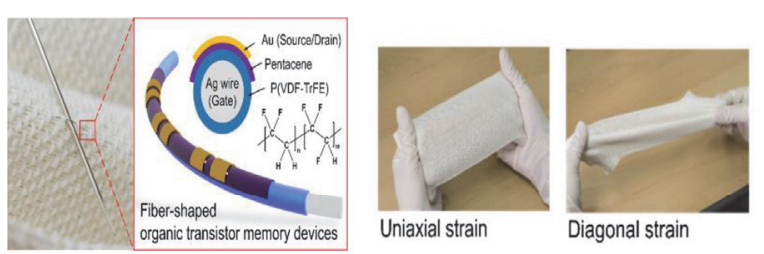

(b)
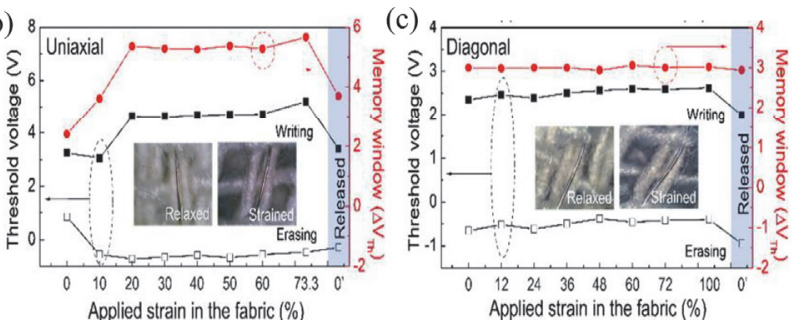

图 4 可拉伸记忆存储晶体管

Figure 4 The stretchable memory transistors

(a) Schematic device structure of the fiber-shaped organic memory transistors. $(b, c)$ Variation of threshold voltage and memory window under different applied uniaxial strains (b) and diagonal strains (c) and at a released state ${ }^{[36]}$. Reprinted with permission from Ref. 36 Copyright (2019) American Chemical Society.

目前来看, 基于传统铁电存储的 STOTs 依赖于两种 稳定极化状态, 而浮栅型记忆存储晶体管是通过将载流 子存储或释放在栅极或绝缘数据存储层的缺陷中进行 记忆 ${ }^{[37]}$, 因而是发展多级存储和稳定性器件的有效途 径. Guo 等 ${ }^{[33]}$ 使用 $\mathrm{p}$ 型聚合物半导体 PCDTPT 设计出一 种基于垂直结构的超短沟道(纳米级)浮栅晶体管. 他们 使用浮栅纳米粒子作为量子点可捕获并存储电荷, 同时 该器件也能在 $V_{\mathrm{G}}=0$ 时刻作为电子读出器. 此外, 浮栅 纳米粒子量子点可有效促进光生激子的分离, 因而器件 还表现出光电子存储特性. 它具有高达 $10^{4} \mathrm{~A} / \mathrm{W}$ 的响应 率、 $73 \mathrm{~V}$ 的存储窗口以及 6 个不同级别存储, 并且在不 同弯曲半径 $(54 \mathrm{~mm}, 10 \mathrm{~mm})$ 下的测试中, 显示出多级光 电子存储器的特性.

\section{4 基于晶体管的传感器}

对于下一代可穿戴和可植入器件而言, 基于 STOTs 的传感器具有信号放大、大面积制备、高机械性能和易 于整合等显著的优点. 目前已有大量文献报道了该类传 感器, 它们不仅可以提供稳定的电力输出, 而且通常不 受其他无关变量的影响.

温度是人体和环境检测中一个重要的检测参数. Ha
等 ${ }^{[38]}$ 开发了一种可拉伸浮栅场效应晶体管构成的温度 灵敏传感器阵列。他们使用聚 $(n$-异丙基丙烯酰胺)/聚 (3,4-乙基二氧噻吩)掺杂聚苯乙烯磺酸盐(PEDOT:PSS) 和热致变色染料涂覆的金 $(\mathrm{Au})$ 电极制备的传感器, 在 $25 \sim 45{ }^{\circ} \mathrm{C}$ 温度下的灵敏度高达 $6.5 \% /{ }^{\circ} \mathrm{C}$. 同时, 涂覆在 栅极上的染料会发生颜色改变, 使温度变化可视化, 此 外该传感器在 $50 \%$ 双轴拉伸下具有稳定的电学性能.

虽然理想的传感器只对预期的测量因子敏感, 但实 际的可拉伸传感器由于外界参数影响往往会呈现出交 叉敏感. Bao 等 ${ }^{[39]}$ 开发出了一种基于单壁碳纳米管 (SWCNTs) 的本征可拉伸温度传感器, 使用了静态和动 态差示法抑制了与应变相关的误差，并实现了高的准确 性和稳定性. 在 $0 \sim 60 \%$ 的单轴应变下, 其输出的绝对 误差为 $\pm 1{ }^{\circ} \mathrm{C}$. 此外, 在最近的报道中他们使用 DPP 基聚合物半导体制备的本征可拉伸温度传感阵列也成 功实现了在 $30 \%$ 单轴应变下, 测量准确度 $<1{ }^{\circ} \mathrm{C}^{[40]}$.

此外, 这种基于 STOTs 实现传感功能的方法, 有望 成为制备可拉伸磁性传感器的有效途径. Zhu 等 ${ }^{[41]}$ 结合 氧化铁纳米粒子 $\left(\mathrm{Fe}_{3} \mathrm{O}_{4} \mathrm{NPs}\right)$ 、银纳米线 $(\mathrm{Ag} \mathrm{NWs})$ 和聚二 甲基硅氧烷(PDMS)优异的磁性、导电性和柔性的特点, 制备了一种可作为功能化磁性电极的复合材料. 经测试 其在 30\%的拉伸应变下电阻只略微上升, 对使用性能几 乎没有影响. 同时, 他们开发了一个基于悬浮栅 STOTs 的磁性传感器, 其具有优异的磁性和机械可调性, 使器 件具有高灵敏度的磁性响应特性. 它的灵敏度高达 $115.2 \% \mathrm{~m} / \mathrm{T}$, 检测限低至 $500 \mu \mathrm{T}$ 的磁场. 此外, 该传感 器被证明在柔性非接触开关和空间分辨磁成像元件方 面具有很好的应用前景.

基于 STOTs 的传感器件的研究当然并不仅限于以 上几类, 进一步开发基于 STOTs 的触觉传感、化学传感 和生物传感等器件将为可穿戴生物电子器件的应用提 供有力支撑.

\section{5 其他功能性晶体管}

突触具有实现神经元间交流的独特结构, 是生物体 内电信号与化学信号传递的重要元件, 通常负责记忆的 编码和存储工作. 受软体动物弹性突触的启发, Shim 等 ${ }^{[42]}$ 使用聚(3-己基噻吩)(P3HT) 纳米纤维的复合材料, 制备出了具有完全橡胶感的突触型晶体管(如图 5a). 该 突触晶体管表现出与生物突触类似的功能包括兴奋性 突触后电流(EPSC)、配对脉冲易化(PPF)、短期记忆 (STM)、长期记忆(LTM) 和高通滤波特性(如图 $5 \mathrm{~b} \sim 5 \mathrm{f}$ ), 且在当机械拉伸达到 $50 \%$ 时，仍能保持这些特性. Bao 等 ${ }^{[43}$ 通过喷墨打印聚合物和 CNTs 制备了可拉伸突触型 晶体管阵列, 其迁移率高达 $30 \mathrm{~cm}^{2} /(\mathrm{V} \cdot \mathrm{s})$, 且在低至 $1 \mathrm{~V}$ 的工作电压下, 通道电流为 $0.2 \mathrm{~mA} / \mathrm{cm}$. 由于这些器件 使用的是本征可拉伸材料，对沿平行和垂直于沟道方向 的应变不敏感，因而当阵列受到 $20 \%$ 机械应变时，它们 的电性能并没有显著下降. 此外, 这种具有双层电容介 
质的晶体管还可以模拟神经元的突触行为.

高跨导的可拉伸晶体管对于可穿戴设备和软体机 器人的集成非常重要. 可拉伸有机电化学晶体管 (SOECTs) 具有低功耗、生物相容性好、高灵敏性等优点, 可以实现高跨导和信号放大功能. Chen 等 ${ }^{[44]}$ 采用微裂 纹 $\mathrm{Au}$ 和 PEDOT:PSS 导体制备了本征可拉伸 OECTs. 在 $0 \%$ 和 $140 \%$ 应变下分别显示出 $0.54 \mathrm{mS}$ 和 $0.14 \mathrm{mS}$ 的高 跨导. 同时在 1000 次的循环测试中保持了高的跨导和 良好的机械稳定性. 高性能 SOECTs 未来可进一步应用 于仿神经形态计算系统的构建. 此外, Yan 等 ${ }^{[45]}$ 基于仿 生原理开发了一种 SOECTs 的传感器, 可对体液中葡萄 糖水平进行灵敏检测 (检测极限大约为 $1 \mu \mathrm{mol} / \mathrm{L}$ ). 该器 件的全向拉伸能力可达 $30 \%$, 弯曲半径可达 $15 \mathrm{~mm}$, 可 以附着在人手的不同部位并保持性能稳定.

尽管目前功能性 STOTs 已经取得了很多显著的研 究成果, 然而大多相关研究还停留在本征可拉伸材料的 开发及性能提升方面. 为此, 进一步开发高性能的 SOPTs、高稳定性的多级存储 OFETs、多功能传感器件 以及新颖的功能器件是实现 STOTs 应用的关键, 也将为 可穿戴生物电子器件的发展提供有力支撑.

\section{3 可拉伸有机光电器件(STOPDs)}

可拉伸光电器件是可穿戴电子和生物医疗设备中 实现光电转换功能的关键组件. 到目前为止, 可拉伸有 机光电器件已经取得了令人兴奋的进展, 包括发光二极 管、交流电致发光器件、发光电化学电池、电致变色器 件等.

\section{1 发光二极管}

发光二极管具有高量子效率、宽视角的特点，是一 种极具吸引力的电致发光器件. 可拉伸有机发光二极管 (STOLED)也被认为是下一代可穿戴显示和固态照明面 板的明星替代产品. 然而，可拉伸发光二极管的发光效 率和机械稳定性仍受结构、材料及拉伸释放过程的限制. 为此, Sun 等 ${ }^{[46]}$ 开发了一种激光可编程屈曲过程的方法, 实现了可控拉伸释放周期的高性能 STOLED. 该器件在 $70 \%$ 应变下，发光效率高达 $70 \mathrm{~cd} / \mathrm{A}$, 同时在 $100 \%$ 的应 变和超 15000 次拉伸循环中其发光效率仅表现出微小的 波动.

胶体量子点因具有独特的光电子特性, 如宽带隙可 调性和高的光吸收系数等而备受关注. Hwang 等 ${ }^{[47]}$ 使用 Graphene/PEDOT:PSS 电极, 通过预拉伸方法实现了一 种基于胶体量子点的高性能可拉伸发光二极管，包括 红、绿、蓝三种(如图 6a). 其中红色 OLED 的峰值亮度 和发光效率分别为 $1310 \mathrm{~cd} / \mathrm{m}^{2}$ 和 $2.25 \mathrm{~cd} / \mathrm{A}$. 该器件可以 在拉伸应变 $70 \%$ 、弯曲曲率半径 $35 \mu \mathrm{m}$ 和 100 次拉伸循 环过程中, 保持其稳定的电学和光学性能(如图 $6 \mathrm{~b}, 6 \mathrm{c}$ ). 这种基于量子点在宽带隙范围内最大限度地提高光敏 性的方法为实现高性能 STOLED 提供了一个极有前景 的发展途径.

\section{2 交流电致发光器件}

交流电致发光器件(ACEL) 是通过场致激发发光, 在发光器件中具有结构简单、低成本和易集成的优势. 可拉伸有机 ACEL 更是在可穿戴、生物医学、智能照 明和显示等方面极具应用潜力. (a)

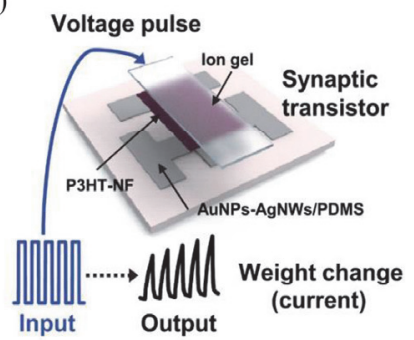

(b)

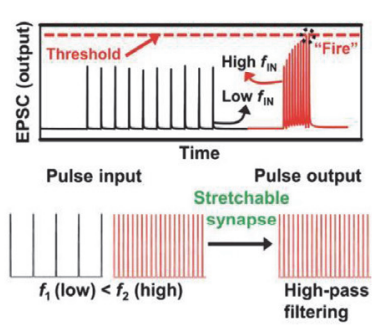

(c)

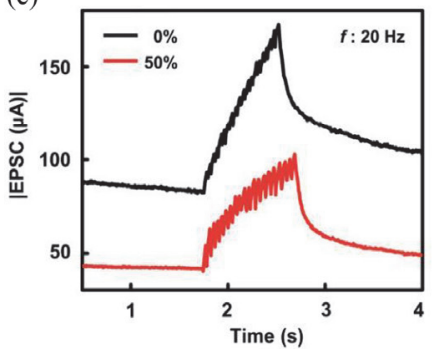

(d)

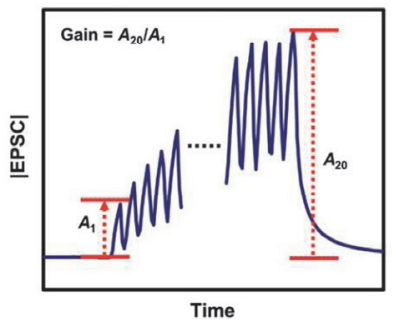

(e)

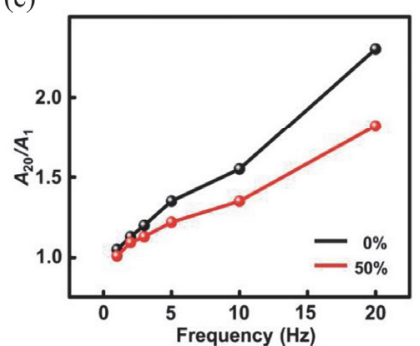

(f)

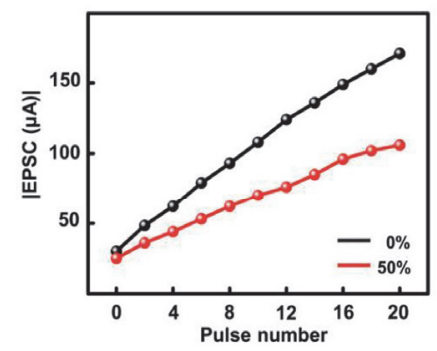

图 5 可拉伸突触型晶体管

Figure 5 The stretchable synaptic transistors

(a) Schematic device structure of the fully rubbery synaptic transistors. (b) Demonstration of high-pass filtering behavior of the stretchable synapse. (c, d) Under $0 \%$ and 50\% strain, the response of measured EPSCs (c) and the definition of the gain $\left(\mathrm{A}_{20} / \mathrm{A}_{1}\right)(\mathrm{d})$ with 20 successive presynaptic pulses at $20 \mathrm{~Hz}$. (e, f) Variation of the gain $\left(A_{20} / A_{1}\right)$ vs various pulse frequencies (e) and the peak current of the EPSC vs various pulse number (f) for the rubbery synaptic transistor with $0 \%$ and $50 \%$ strain ${ }^{[22]}$. Reprinted with permission from Ref. 42 Copyright (2019) AAAS. 
(a)

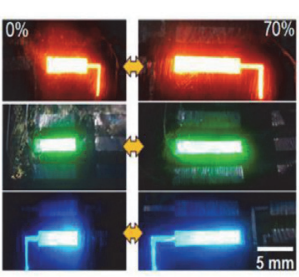

(b)
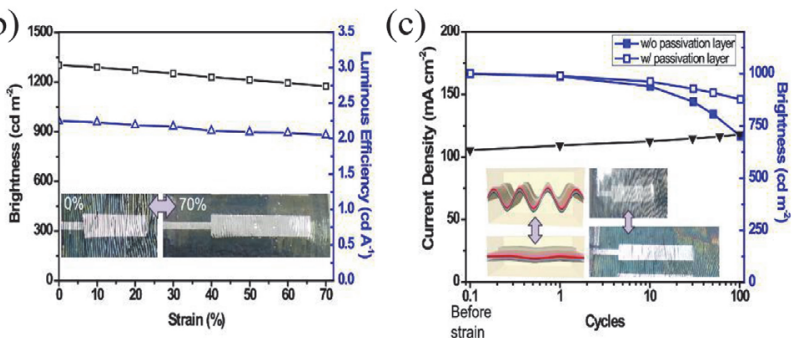

图 6 可拉伸发光二极管

Figure 6 The stretchable lights-emitting diodes

(a) Photographs of the stretchable QD-LEDs with three different color under $0 \%$ and $70 \%$ strain. (b) Brightness characteristics and luminous efficiency characteristics of the stretchable red QD-LED with $0 \%$ and $70 \%$ strain. (c) The relationship of current density and brightness with strain cycles under $0 \%$ and $70 \% \operatorname{strain}^{[47]}$. Reprinted with permission from Ref. 47 Copyright (2017) American Chemical Society.

在工艺方面, Meng 等 ${ }^{[48]}$ 采用低成本和全溶液的工 艺制备了一种纤维状可拉伸的 ACEL, 其具有良好的拉 伸性能、发光性能、稳定性以及近乎零的迟滞. 该器件 在 $50 \%$ 应变的情况下, 在 6000 多次拉伸释放循环中仍 能保持稳定的亮度.

在高机械性能方面, Shan 等 ${ }^{[49}$ 报告了一个由摩擦电 纳米发电机驱动的本征可拉伸和透明 ACEL, 他们将铜 包埋的 PDMS 和 SWCNTs 组成的两个电极夹在一起, 形 成了电致发光层. 该器件在拉伸应变达到 $100 \%$ 时, 无 论是在准静态还是动态变形下均能表现出明亮、持久的 电致发光.

在大面积集成阵列制备上, Ahn 等 ${ }^{[50]}$ 使用一种将 Graphene 和 Ag NWs 嵌入 PEDOT:PSS 薄膜的混合电极, 开发出了由可拉伸 ACEL 器件构成的 $8 \times 8$ 无源阵列. 该阵列在高强度的机械变形情况下(拉伸、弯曲、卷曲 或折叠)均表现出高效和均匀的电致发光(如图 7).

\section{3 其他光电器件}

有机发光电化学电池(OLECs) 是使用有机发光材料 作为活性层, 在电子和离子混合电导基础上实现电致发 光的电化学器件. Pei 等 ${ }^{[32]}$ 使用 $\mathrm{Ag} \mathrm{NWs-}$ 聚氨酯丙烯酸 酯复合电极制备的聚合物 STOLECs 在室温下表现出橡 胶般的弹性, 具有可折叠性, 可在重复的连续拉伸循环 中稳定工作. 此外, 他们还发现器件的发光亮度随应力 增加先升高再降低. 而器件的发光效率也在 $40 \%$ 应力下 从 $1.0 \mathrm{~cd} / \mathrm{A}$ 提高到 $3.0 \mathrm{~cd} / \mathrm{A}$, 在达到 $80 \%$ 时趋于稳定然 后开始下降，但在 $120 \%$ 时仍保持比初始值高出 $100 \%$ 的 发光效率 $(2.1 \mathrm{~cd} / \mathrm{A})$. 研究表明这可能由于小的拉伸使 电子和空穴注入更加平衡, 从而可以显著提高其发光 (a)

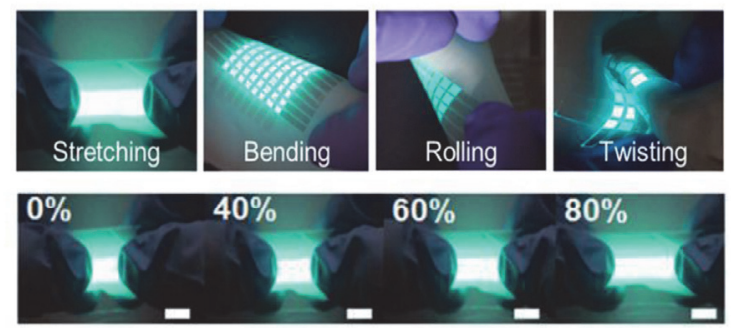

(b)
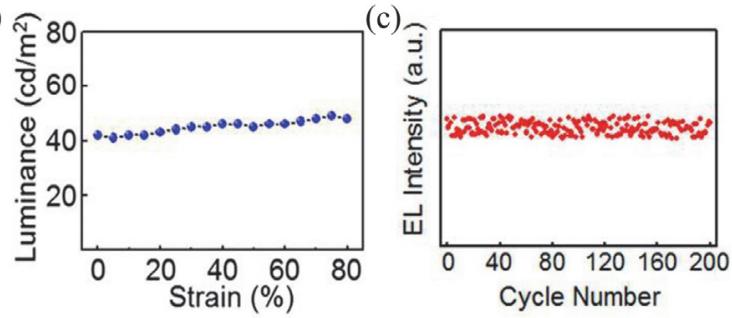

图 7 可拉伸交流电致发光器件

Figure 7 The stretchable alternating-current electroluminescent devices (a) The array under bending, rolling, twisting and stretching with different strain levels. (b, c) Variation of the electroluminescence intensity under different strains (b) and mechanical stability for 200 cycles of the ACEL device (c) ${ }^{[50]}$. Reprinted with permission from Ref. 50 Copyright (2019) American Chemical Society.

效率. 尽管可拉伸 OLECs 在近几年来取得了一定进步, 然而其切换速度和长期稳定性仍然需要改进. 特别是, 该类器件的性能高度依赖于电极的电导率, 而电导率直 接影响着注入电流, 这也是高度应变下实现 OLECs 性 能稳定的最大限制因素 ${ }^{[51]}$.

高介电常数 $(\kappa)$ 栅介质发光电容器(HLEC) 通过将发 光介质层并入弹性体并夹在两个可拉伸电极之间，极其 巧妙地实现了光发射和传感功能的整合. Shepherd 等 ${ }^{[52]}$ 制备了一种基于水凝胶的 HLEC, 它可以在拉伸到 $>480 \%$ 的橡胶薄层上进行光发射和触摸传感. 通过利 用改变电极面积和距离会改变电容的原理, 实现了对压 力和拉伸变形的双重感应. 此外, 当这些薄片被集成到 软体机器人的皮肤中时, HLEC 阵列可提供动态着色和 对内外刺激的反馈. 此外, Tee 等 ${ }^{[53]}$ 基于 HLEC 开发了一 种透明、高介电常数聚合物介质的低场照明光电可拉伸 器件装置. 该设备的交流电压为 $23 \mathrm{~V}$, 频率低于 $1 \mathrm{kHz}$, 同时在 $2.5 \mathrm{~V} / \mu \mathrm{m}$ 稳定照明时亮度为 $1460 \mathrm{~cd} / \mathrm{m}^{2}$, 最大应 变为 $800 \%$. 虽然该器件的光视效能目前无法超越商业 ACEL，但已经取得了巨大进步.

电致变色技术是一种可以通过电流控制来调节光 学性质的非发射信号技术. Bao 等 ${ }^{[54]}$ 基于压力传感器和 有机电致变色器件(OECDs)开发出了一种具有交互的颜 色变化和触觉传感的可拉伸电子皮肤. 该电子皮肤通过 改变施加的压力和施加压力的持续时间, 可以很容易地 控制电子皮肤的颜色. 这里, 他们使用 P3HT(中性时呈 暗红色, 氧化时呈淡蓝色)作为电致变色层制备出了完 全可拉伸、透明、轻便的 OECDs. 
目前来说, 研究人员已经在可拉伸有机光电材料与 器件方面进行了诸多开拓性的工作. 然而, 发展本征可 拉伸有机光电器件仍面临着诸多阻碍, 包括 OLED 器件 较低的发光效率和差的机械稳定性、ACEL 器件难以权 衡的发光特性与透明度、可拉伸性以及传统光电器件的 材料限制等. 未来发展高性能的本征可拉伸有机光电器 件不仅可以满足现实应用的需求, 还能使生物医学成 像、曲面显示器和电子报纸等大量新应用成为可能.

\section{4 可拉伸有机能源存储和转换器件(STOEDs)}

可拉伸能源存储和转换器件即使在高机械强度下 也能稳定供能, 因而对可穿戴移动电源的发展至关重 要. 可拉伸有机能源存储和转换器件具有优异的机械性 能、生物相容性以及可与人体自然贴合的特点, 在基础 设施、医疗、安全和通信行业等领域受到了越来越多的 关注. 随着可拉伸导体和电极的发展趋于成熟, 可拉伸 有机能量转换和存储系统, 包括太阳能电池、超级电容 器以及纳米发电机等, 出现了许多重要的研究成果.

\section{1 太阳能电池}

太阳能电池作为一种非常有前途的能源器件, 受到 了人们的广泛关注. 虽然无机太阳能电池通常表现出很 高的效率, 但可拉伸有机太阳能电池(OSCs)作为下一代 可穿戴的电源设备更具有吸引力. 然而, 较低的抗屈曲 能力限制了 OSCs 的商业应用. 最近, Sun 等 ${ }^{[55]}$ 将简单的 模板转移技术应用于基于聚合物的可拉伸 OSCs, 实现 了一种有序的屈曲轮廓. 值得注意的是, 周期性搭扣的 形成有效提升了器件性能, 其中拉伸应变为 $100 \%$, 光 电转换效率为 $5.1 \%$, 开路电压为 $0.78 \mathrm{~V}$, 短路电流密度 为 $12 \mathrm{~mA} / \mathrm{cm}^{2}$, 填充因子 $(\mathrm{FFs})$ 为 $54 \%$. 该器件在数万次 的拉伸释放循环测试中也表现出稳定的性能.

Pei 等 ${ }^{[29]}$ 使用二碘辛烷(DIO)及聚([3,4- $\left.b\right]$-噻吩/苯二 噻吩)(PTB7)和亚甲基富勒烯的给-受体共混物, 开发出 了一种固态本征可拉伸的 OSCs(如图 8a). 该器件呈半 透明状，在室温下表现出橡胶弹性. 同时在 $100 \%$ 应变 下, 晶粒之间的相对滑动实现了纳米晶粒和 PTB7 聚合 物链的整体取向移动，而这种取向也是可逆的. 在 $50 \%$ 的压力下拉伸, 测得的功率转换效率(PCE)为 $3.48 \%$, 拉 伸 1 个周期后, $\mathrm{PCE}$ 提高到 $3.67 \%$, 这是由于当电池被拉 伸时, 有效面积的扩大使器件的总发电量显著增加. 此 外, 在 100 次循环拉伸/释放测试中该器件的 PCE 值仅 略微下降, 但仍能保持到 $2.99 \%$, 同时其电学性能也没 有显著降低(如图 $8 \mathrm{~b} \sim 8 \mathrm{~d}$ ).

\section{2 超级电容器}

超级电容器(SCs)的能量密度虽无法与电池相謧美, 但能提供更高的功率密度, 特别在高功率的突发系统 上. 此外, 可拉伸 SCs 在高度机械形变下还可以维持稳 定的充放电循环, 是非常有前途的有机能源存储器件. (a)

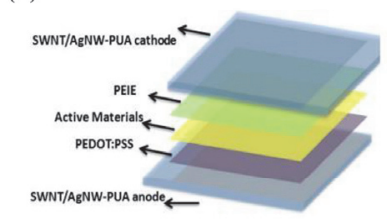

(c)

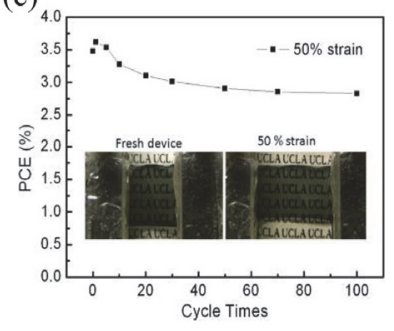

(b)

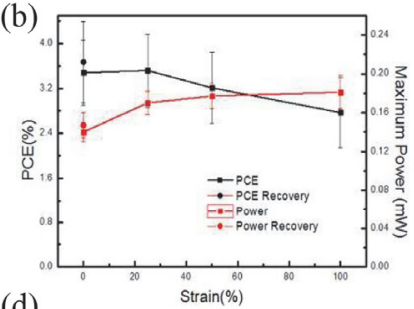

(d)

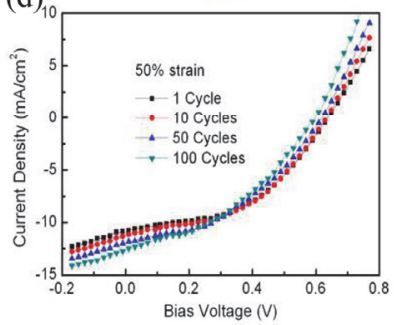

图 8 可拉伸有机太阳能电池

Figure 8 The stretchable organic solar cells

(a) Schematic diagram of the sandwich structure of the stretchable OSCs. (b) Output power and PCE of the OSCs under various strains. (c) Variation of the PCE after stretching-relaxing cycles, and (d) $J-V$ characteristics of the OSCs under $50 \%$ strain ${ }^{[29]}$. Reprinted with permission from Ref. 29 Copyright (2017) American Chemical Society.

虽然通过不同的材料设计和组合策略, 可拉伸超级 电容器取得了可喜的进展, 但是在具有多种功能的薄层 结构中实现高度柔性的 SCs 并非易事. Cheng 等 ${ }^{[56]}$ 通过 自组装垂直 $\mathrm{Au} N \mathrm{~N}$ s 和电沉积聚苯胺(PANI), 开发了一 种皮肤状的电致变色超级电容器. $\mathrm{Au} \mathrm{NWs}$ 薄膜具有高 导电性, 同时展现出良好的柔韧性和变形能力, 而 PANI 在提高电容和充放电电平指示器方面具有双重作 用. 所组装的 SCs 具有优异的一致性, 其面积比电容可 达 $11.76 \mathrm{mF} / \mathrm{cm}^{2}$ (扫描速率为 $10 \mathrm{mV} / \mathrm{s}$ ), 并具有很高的 稳定性. 该可穿戴纹身能量装置可以在多种手部运动和 皮肤变形条件下保持稳定的电学性能.

传统电解质的 SCs 通常不具有本征可拉伸性和自 愈性能, 难以满足实际应用中高机械形变的需求. 据此, Zhi 等 ${ }^{[57]}$ 开发了一种通过氢键交联聚丙烯酸和乙烯基杂 化二氧化硅纳米颗粒(VSNPs-PAA)的新型电解质, 提供 了一个制备自愈性和高拉伸性 SCs 的有效方法. 该器件 在 500 次断裂/愈合循环测试中呈现出 $100 \%$ 自愈, 此外 其在 $600 \%$ 的应变下性能有一定增强.

微超级电容器(MSCs)具有小型化易整合、超高的功 率密度和高频响应的特点, 是能源存储器件的优秀候选 者. Lee 等 ${ }^{[58]}$ 设计和制造了一个基于还原石墨烯氧化物 $(\mathrm{rGO}) /$ 金异质结构模式的可拉伸 MSCs. 该器件通过高 重复率飞秒激光脉冲实现了由垂直方向异质结构组成 的相互连接、稳定的三维网络. 在转移到 PDMS 后, 3D 网络获得了约 $10^{5} \mathrm{~S} / \mathrm{m}$ 的高电导率, 即使在 50\%的应变 下, 电导率也能保持在约 $10^{4} \mathrm{~S} / \mathrm{m}$.

\section{3 纳米发电器件}

纳米发电机(NGs)因可以将机械能、振动能和水力 能转换为电能而备受关注. 可拉伸有机纳米发电机 
(SONGs) 实现了对小尺度器件的供电, 在自供电系统的 发展中起着至关重要的作用.

压电纳米发电机(PENGs)是柔性电子器件的理想电 源, 适合于自供电的穿戴式传感系统. Lee 等 ${ }^{[59]}$ 基于可 拉伸石墨电极和压电纳米纤维叠片制备了一种高效的、 全方位可拉伸的 PENGs. 压电敏感层是由嵌入钛酸钡 纳米颗粒的 PU 和聚(偏氟乙烯-三氟乙烯)复合纳米纤维 组成. 由于衬底上的 $3 \mathrm{D}$ 微图案和独立堆叠的复合纳米 纤维的应力消除特性, 该器件具有高达 $40 \%$ 的拉伸能 力, 且在 $30 \%$ 应变下具有高达 9000 个拉伸循环的机械 耐久性. 此外, 该器件的峰值开路电压 $\left(V_{\mathrm{oc}}\right)$ 和短路电流 $\left(I_{\mathrm{sc}}\right)$ 分别是 $9.3 \mathrm{~V}$ 和 $189 \mathrm{nA}$, 还可以附在受试者膝盖骨 上从人体运动中获取能量.

摩擦电纳米发电机(TENGs) 是基于接触带电和静电 感应的耦合效应, 可将机械能转化为电能. TENGs 作为 一种低成本、易加工、材料广泛的能量转换装置, 可广 泛应用于多种能源的收集. Wang 等 ${ }^{[60]}$ 报告了一种超可 拉伸的皮肤状的 TENGs, 它通过将弹性材料和离子水 凝胶分别作为带电层和电极, 实现了生物力学能量的收 集和触觉感知. 首次实现了能量收集装置同时兼具超高 的可拉伸性(单轴应变 $1160 \%$ )和透明度(可见光平均透 过率 $96.2 \%$ ). 该器件输出的交流电瞬时功率密度峰值可 达到 $35 \mathrm{~mW} / \mathrm{m}^{2}$, 能成功驱动可穿戴电子设备.

开发新型可拉伸 NGs 可为多功能电源和可穿戴电 子领域的潜在应用提供新机遇. 受电鳗细胞膜上离子通 道结构的启发, $\mathrm{Li}$ 等 ${ }^{[61]}$ 使用硅烷-PDMS 复合材料、聚四 氟乙烯(PTFE)和聚乳酸(PLA)等制备了一种可拉伸仿生 纳米发电机(BNGs), 用于水下能量收集(如图 9a, 9b).
该仿生器件可以从水下人体运动中获取机械能, 并输出 $10 \mathrm{~V}$ 以上的开路电压，成功应用于人体运动位置监测 和水下救援系统. 此外, 该器件还具有良好的柔韧性、 可拉伸性 $(>60 \%)$ 和抗拉疲劳性能(抗拉疲劳强度超过 5 万次)等优点, 是一种很有前途的水下可穿戴电子产品 的可持续电源(如图 9c, 9d). 此外, 无论是 PENGs、 TENGs 还是 BNGs 均具有一定的力学敏感性, 这使其还 可作为人工电子皮肤应用于压力/触觉感知.

近年来, 基于聚合物电解质的研究迅速发展起来, 同时有关结构工程的可拉伸能源器件也已经做了大量 的工作. 然而, 其复杂的制造工艺、高成本和低拉伸性 能制约了这些器件潜在的应用. 此外, 可拉伸有机能源 器件目前仍存在着电学性能低和稳定性差等问题. 因此 发展高拉伸性、高机械稳定性和自主愈合的可拉伸能源 存储和转换器件是未来实现自供能电子设备商业应用 的必要条件, 也是开发下一代能源器件的关键.

\section{5 可拉伸有机传感器(STOSs)}

可拉伸传感器是通过信号转换进行数据读取, 可贴 附于人体组织, 实现人与器件的自然结合. 可拉伸有机 传感器具有高灵敏性、高拉伸性和透明性, 是检测外界 信号的理想平台. 随着技术的发展, 可拉伸有机传感器 的开发使智能仿生电子设备, 包括但不限于软体机器 人、假肢等新兴领域的潜在应用成为可能 ${ }^{[62]}$. 近年来, 包括压力、应变、触觉、温度、气体等大量传感器的研 究成果共同促进了可拉伸有机传感器的发展, 使得这种 低成本、可大面积制备的电子皮肤技术应用成为可能. (a)

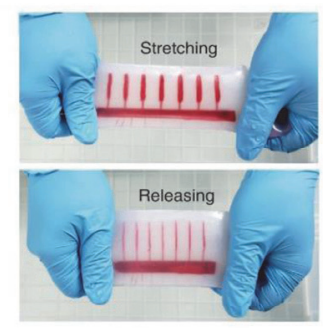

(b)

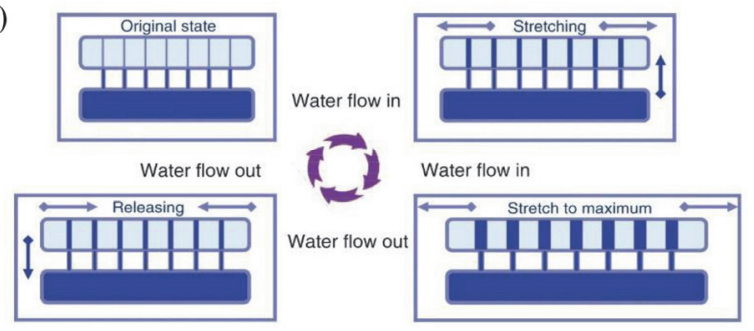

(c)

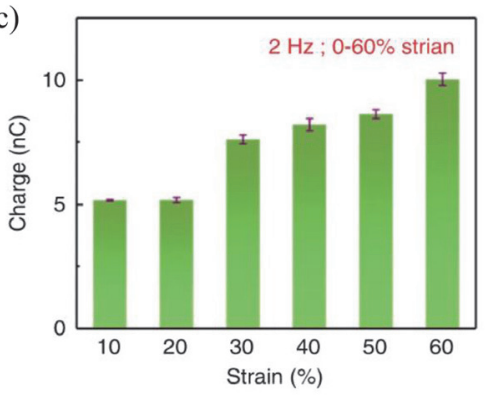

(d)

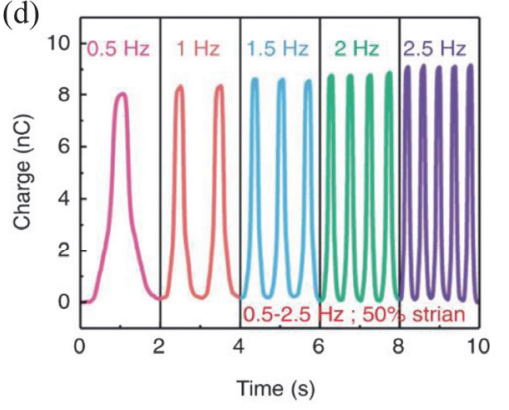

图 9 仿生可拉伸纳米发电机

Figure 9 The bionic stretchable nanogenerator

(a) Photographs of BNGs and (b) schematic diagram of the working process of BSNG. (c, d) $Q_{\text {sc }}$ (right) of BSNG under various strain (stretched by a linear motor, at $2 \mathrm{~Hz}$ ) (c) and under various frequency with $50 \%$ strain (d) ${ }^{[61]}$. Reprinted with permission from Ref. 61 Copyright (2019) Springer Nature. 


\section{1 压力和应变传感器}

机械传感器通常是通过一些转换原理(如电容、压 阻、压电和摩擦电)来检测机械变化 ${ }^{[63]}$. 在这些传感器 中, 压阻传感器和电容传感器是最常用的, 因为它们的 设计简单, 并且能够检测静态和动态信号.

基于几何结构工程设计的可拉伸传感器是实现压 力和应变传感的有效途径, 近年来相关研究也取得了许 多显著成果. Zhang 等 ${ }^{[64]}$ 将 CNTs/Graphene 和微结构聚 二甲基硅氧烷(m-PDMS)分别作为活性材料和柔性基体, 制备了一种基于仿生分层结构和高导电活性膜的高性 能压力传感器. 由于独特的层次结构, 所制备的压力传 感器表现出高灵敏度 $\left(19.8 \mathrm{kPa}^{-1},<0.3 \mathrm{kPa}\right)$ 、低检出限 $(0.6 \mathrm{~Pa})$ 、快速响应时间 $(<16.7 \mathrm{~ms})$ 、低工作电压 $(0.03 \mathrm{~V})$ 以及超过 35000 次循环的稳定性能. Zheng 等 ${ }^{[65]}$ 针对高 灵敏应变传感器的工程高拉伸性能, 提出了一种簇状微 结构策略(如图 10a). 该微结构在拉伸过程中从紧密排 列到有序间隔状态的变形决定了应变传感器的电阻变 化, 从而实现了相当高的器件性能, 包括高灵敏度(测 量因子高达 2700) 和高拉伸性(应变传感范围可至 160\%)(如图 10b, 10c). (a)

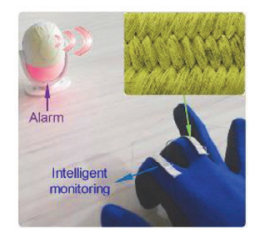

(c)

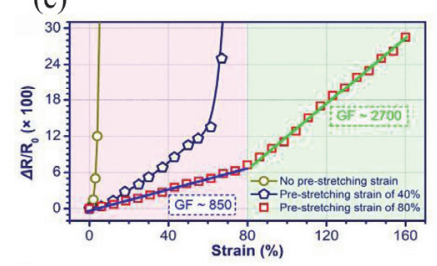

(b)
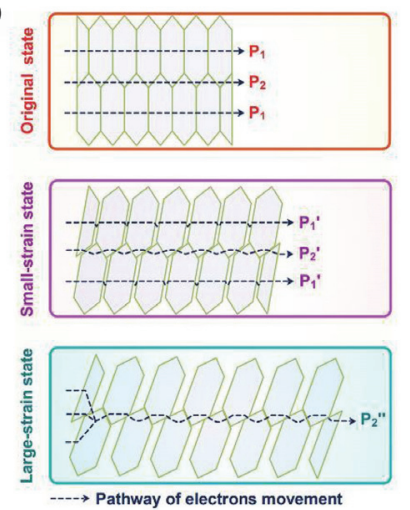

图 10 可拉伸应变传感器

Figure 10 The stretchable strain sensor

(a) Photograph of triggering commands with two fingers via the strain sensors to achieve the intelligent control of an audible/visual alarm. (b) Schematic diagrams of the strain sensor with various stretched states. (c) Change in the resistance of the strain sensor under $0 \%, 40 \%$, and $80 \%$ pre-stretching strain $^{[65]}$. Reprinted with permission from Ref. 65 Copyright (2019) American Chemical Society.

开发多功能可拉伸传感器对于可穿戴设备而言非 常重要. 然而目前的传感器大多是单一响应, 同时检测 压力和应变的传感器尚待研究. 据此, Zhang 等 ${ }^{[66]}$ 采用 两条具有不同弯曲或受压电阻的正交碳纳米管-聚氨酯 海绵带(CPSSs), 制作出一种能够独立检测全向弯曲和 压力的多功能可拉伸传感器. 基于带材形状在不同方向 弯曲时阻力变化不同的特征, 两个相互垂直的 CPSSs 可 以同时反映弯曲距离和弯曲方向的信息(误差可控制在 $4 \%$ 以内). 同时借助摩擦电效应, 该器件能有效区分弯
曲和压力，保证了设备在复杂情况下工作.

可植入器件通常对生物相容性有着严格的要求，同 时在使用时为避免二次手术伤害也亟需可降解器件的 应用. Bao 等 ${ }^{[67]}$ 报道一种完全由生物可降解材料制成的 可植入压力应变传感器. 它可以使用两个垂直隔离的传 感器独立地测量应变和压力, 这两个传感器能够分辨出 $0.4 \%$ 的应变和 $12 \mathrm{~Pa}$ 的压力, 而不会相互干扰. 该器件 迟滞很小, 且响应时间在毫秒范围内, 具有良好的循环 稳定性. 此外, 其在大鼠体内研究中显示出良好的生物 相容性和功能.

\section{2 触觉传感器}

触觉感知是软体机器人的关键技术之一, 它能够识 别振动和短暂接触的瞬间. 可拉伸有机触觉传感器凭借 大面积、极薄、符合曲面的优势, 实现了从机器人到医 疗设备再到可穿戴电子的全面应用.

近年来，如碳纳米管、石墨烯等高性能材料的应用 有效地提高了触觉传感器的各项性能. Bayer 等 ${ }^{[68]}$ 以丁 腈橡胶为模板, 通过将分散纳米碳纤维(CNF)或石墨烯 纳米片(GNPs) 的导电聚合物悬浮液喷涂在橡胶片两侧, 开发出一种具有触觉传感特性的可拉伸电容式传感器. 该方法可以降低电极表面电阻至约 $10 \Omega / \mathrm{m}$, 同时由重 复延伸/恢复周期引起的结构损伤也可以通过退火得到 修复. 此外, 该器件的触觉感知范围为 $0.03 \sim 5 \mathrm{~N}$, 且在 拉伸或弯曲表面上很容易测到, 表明了其具有良好的机 械性能和灵敏度.

除了新材料的使用，引入不同的物理传导机制也将 促进高性能触觉传感器的发展. 其中基于麦克斯韦位移 电流的摩擦电纳米发电机(TENG)感应技术发展尤其迅 速. Pan 等 ${ }^{[69]}$ 开发出了一个自供电、透明和高可拉伸性 的摩擦电触觉传感器. 此外还制备了一个 $8 \times 8$ 的摩擦 电传感器矩阵, 该矩阵在高应变 $(>100 \%)$ 条件下由于静 电感应的作用可以良好工作。同时使用交叉定位技术， 该设备可以致动并更快速的实现触觉映射, 响应时间为 $70 \mathrm{~ms}$ ，被检测物可以是任意常用材料和人.

传感信号的可视化是传感器件发展的最终要求. 受 变色龙启发, Bao 等 ${ }^{[54]}$ 基于电致变色半导体 P3HT 开发 出了一种具有触觉敏感的可拉伸电子皮肤(如图 11a). 该电子皮肤通过施加压力的改变和持续时间, 可以很容 易地控制电子皮肤的颜色(如图 11b,11c). 该器件通过 将触觉传感器与电致变色装置的集成, 使低功耗、互动 性和可变色的可拉伸电子皮肤的演示成为可能. 此外, Wang 等 ${ }^{[70]}$ 基于两个串连的发光二极管(LED)设计了一 个简单的触觉传感单元. 他们利用人体或其他介质接触 实现了传感电路与电磁波间感应效应的放大. 而在电磁 信号的激发下, LED 的指数特性导致电流信号的增强. 因此, 通过观察亮度的变化可以检测接触点电磁信号情 况，从而使接触行为可视化. 
(a)

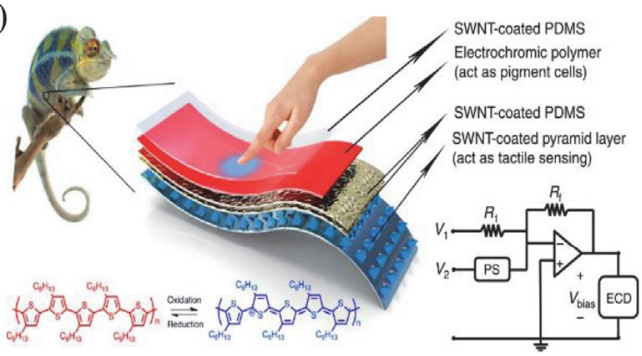

(b)

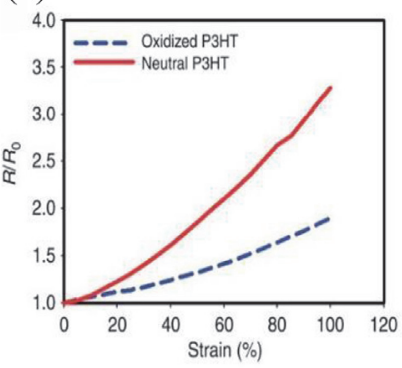

(c)

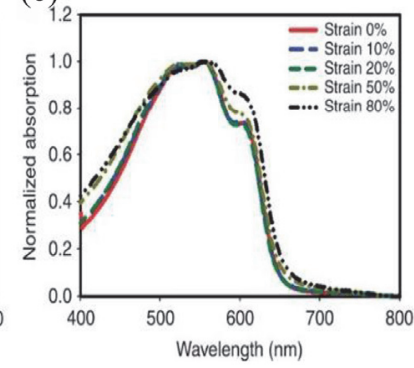

图 11 可拉伸触觉传感器

Figure 11 The stretchable tactile sensor

(a) Schematic diagram of the chameleon-inspired circuit layout with tactile sensor and electrochromic devices. (b) Mechanical characterization and (c) absorption spectra of P3HT ECDs under various strains ${ }^{[54]}$. Reprinted with permission from Ref. 54 Copyright (2015) Springer Nature.

\section{3 温度传感器}

温度感知是人体皮肤感觉系统的一项关键功能, 它 可以迅速灵敏地探测外部环境的信息. 近年来, 可拉伸 有机温度传感器不仅仅集中于使用场效应晶体管和电 阻式传感技术, 还发展出许多新的设计策略和成果.

基于几何结构设计的传感器已经被证明是实现可 拉伸温度传感的成熟方法. 其中, 纤维状器件可以很好 地附着在织物上并应用于可穿戴器件, 吸引了广泛关 注. Lee 等 ${ }^{[71]}$ 使用 rGO/PU 复合材料开发出了一种可拉伸 温度传感纤维. 该器件采用蛇形结构消除了应变诱导的 干扰, 使应变不敏感的可拉伸温度传感器得以实现. 该 器件可通过控制 $\mathrm{rGO}$ 的还原时间来调节热响应指数, 这 是由于含氧官能团密度的变化会影响跳跃电荷输运和 过量载流子的产热. 此外, 该器件也表现出较好的机械 性能和传感特性，包括高达 $90 \%$ 的可拉伸性、 $0.8 \% /{ }^{\circ} \mathrm{C}$ 的 响应率以及约 $0.1{ }^{\circ} \mathrm{C}$ 的传感分辨率，同时其在 $0 \sim 50 \%$ 应变范围内还具有 $\pm 0.37{ }^{\circ} \mathrm{C}$ 的响应稳定性. 事实上, 它 可以连续检测人体运动过程中皮肤温度的变化.

具有门控器件结构的透明、可拉伸(TS)传感器, 其 材料本质上是透明、可拉伸的, 因而不需要进行结构工 程. Lee 等 ${ }^{[72]}$ 将温度灵敏的 rGO 纳米薄片插入弹性 PU 基体中形成的复合材料作为温度传感层开发出了全弹 性体的 TS 电阻和 TS 门控温度传感器阵列. 该温度传感 器的最大应变为 $70 \%$ 、灵敏度高达 $1.34 \% /{ }^{\circ} \mathrm{C}$, 且在 $30 \%$ 应变下经过 10000 次拉伸循环后仍能保持其响应. 此外, 该器件能够检测到低至 $0.2{ }^{\circ} \mathrm{C}$ 的温度变化, 并对温度变

化的响应非常快，可以方便、准确地附着在物体或人体 上, 实现表面温度的监测.

\section{4 其他传感器}

在环境和健康等监测中, 气体传感器的重要性不言 而喻. 然而被测气体中通常存在一些干扰因素比如湿 度、杂质等会影响器件的传感性能. 据此, Liu 等 ${ }^{[73]}$ 在弹 性祄底上以柔性石墨烯为电极制备了一层皱褶量子点 (QD)传感层, 实现一个完全可拉伸和抗湿度的气体传 感器. 通过对可拉伸基底预应变的控制，可以使折叠后 QD 薄膜呈均匀的波形结构, 使其抗湿度干扰性能得到 改善. 这是由于所采用的胶体量子点具有优异的溶液可 加工性, 为器件提供了多自由度. 该器件不仅具有较高 的灵敏度和可拉伸性，且具有良好的稳定性，在室温下 实现了 $\mathrm{NO}_{2}$ 高响应. 即使在 1000 次拉伸/释放循环测试 中也能达到稳定工作.

随着生物和智能电子领域的应用, 电生理传感器吸 引了人们的兴趣. Park 等 ${ }^{[74]}$ 开发了一种基于葡萄糖传感 器的软的、智能的隐形眼镜. 该传感器的主要机制是通 过葡萄糖氧化酶(GOD)的氧化还原反应实现了对葡萄 糖的选择性和敏感性检测, 在此过程中产生的质子 $\left(\mathrm{H}^{+}\right)$ 会引起石墨烯通道( $(\mathrm{p}$ 型)的正电荷转移效应. 因此, 载流 子密度与葡萄糖浓度成正比, 可以用来检测葡萄糖的浓 度. 该传感器可以检测糖尿病患者眼泪中超过 0.9 $\mathrm{mmol} / \mathrm{L}$ 的平均葡萄糖水平，其灵敏度约为 $22.72 \% /(\mathrm{mmol} / \mathrm{L})$ 、信噪比为 $23.87(0.1 \mathrm{mmol} / \mathrm{L}$ 血糖水平 下)、最低检测浓度低至 $12.57 \mu \mathrm{mol} / \mathrm{L}$ (对应信噪比约 3). 值得注意的是, 该器件在 $30 \%$ 拉伸应变下其响应变化可 以忽略. 此外, 他们将该器件与显示器进行集成所制的 智能透明隐形眼镜, 可以提供监测泪液中的葡萄糖浓度 的实时、无线操作，同时通过匹配局部图案区域的折射 率来提供清晰的视野.

近年来, 基于无机材料的巨磁阻(GMR)和霍耳效应 的磁传感器已取得了一系列的研究成果. 然而构建多功 能和高灵敏度的可拉伸磁传感器件的研究仍然是一个 挑战. 除了已介绍的基于 OFETs 的磁传感器外, 还有一 种基于各向异性磁阻效应(AMR)的磁传感器吸引了人 们的广泛关注. Sun 等 ${ }^{[75]}$ 在聚对苯二甲酸乙二醇酯(PET) 祄底上使用 $\mathrm{Ta}(3 \mathrm{~nm}) / \mathrm{Py}(30 \mathrm{~nm}) / \mathrm{Ta}(5 \mathrm{~nm})$ 作为磁阻层, 开发了一种自偏 AMR 传感器. 通过磁极的设计实现了 在零偏压下, 输出电压与加磁场呈线性变化. 此外, 其 在 $3 \mathrm{~Hz}$ 下检测限约为 $150 \mathrm{nT}$, 同时还具有可与刚性祄 底上 GMR 器件相媲美的灵敏度 $42 \mathrm{~T}^{-1}$. 此外, 该传感 器具有良好的变形稳定性, 对弯曲半径为 $10 \mathrm{~mm}$ 的传感 器也有稳定的电响应 $(\Delta U / U<3.6 \%$ ), 而当弯曲半径为 5 $\mathrm{mm}$ 的传感器释放后, 磁场灵敏度可完全恢复. 因而, 该器件可以附在弯曲手指或手腕周围作为可穿戴设备, 为人类提供额外的磁感应. 
多功能传感器的开发对于实现可穿戴和多功能电 子器件应用具有一定的参考价值. Wang 等 ${ }^{[76]}$ 最近报道 了一个真皮组织启发的高度可拉伸和整合的系统级矩 阵网络(如图 12a). 他们在一个结构化的聚酰亚胺网络 上实现了特定可扩展传感器单元的三维(3D)集成. 该器 件可以同时进行多刺激感知包括温度、面内应变、湿度、 光线、磁场和压力等(如图 $12 b \sim 12 f$ ), 成功地扩展了电 子皮肤的传感功能. 此外, 它还具有可调感知范围和大 面积的特点, 可应用于构建个性化的智能假肢.

以上研究足以表明, 可拉伸有机传感的开发和应用 已取得了巨大的成功. 通过对各项测量因素的监测和设 备的控制也很好地证明了可拉伸有机传感器的应用潜 力. 然而, 目前的研究中有关本征可拉伸的有机传感器 的报道少之又少, 同时真正实现高性能传感的器件也非 常有限. 因此未来的研究应该集中在开发本征可拉伸传 感材料、制备高性能的传感器件和设计新颖传感电路上, 从而满足实际需求进一步拓宽其应用范围.

\section{6 可拉伸有机存储器(STOM)}

在信息时代中, 数据的急速增长带来的是对存储器 性能要求的进一步升级. 传统的数据存储器在遇到机械 应变时易发生断裂，将会造成不可恢复的数据丢失. 可 拉伸有机记忆存储器具有高机械稳定性、低成本、易整 合等优势, 使其在各类存储器中脱颖而出, 成为下一代 非易失性存储器最有前途的候选者. 非易失性有机存储
器包括铁电存储器、浮栅存储器、忆阻器、磁性存储器 以及仿突触存储器等. 其中, 前两种存储器在第一章中 已有介绍, 因而本章主要讨论忆阻器、磁性存储器以及 近年来受到广泛关注的仿突触存储器三种.

\section{1 忆阻器}

忆阻器是基于外加电场变化时引起材料的电导率 相应改变从而进行随机读取存储的一类器件. 可拉伸有 机电阻存储器特别是聚合物存储器具有运行快、功耗 低、结构简单、集成度高等优势，因而是面向可穿戴存 储器件的关键技术 ${ }^{[37]}$.

开发具有良好电学性能和可拉伸的聚合物有源层 是实现可拉伸电阻存储器的基础策略. Chen 等 ${ }^{[77]}$ 通过 将柔性的硅氧烷链引入聚酰亚胺的主链合成出具有良 好拉伸性能的聚(硅氧烷-亚胺) (ODPA-A12), 并以交联 的 PDMS 弹性体为基底、CNTs 为电极, 实现了一种可 拉伸的电阻存储器件. 该器件不仅在 $40 \%$ 应变下具有稳 定的一次写入多次读取(WORM)的记忆特性，且具有高 的开关比 $\left(>10^{6}\right)$, 同时在 $20 \%$ 应变下 600 次可拉伸循环 性能测试中，表现出较高的机械耐久性.

此外, 表面形态一定程度上会影响存储器的电阻开 关特性，裁剪表面形态被证明是制备可拉伸聚合物存储 器的一种简便而通用的方法. Huang 等 ${ }^{[78]}$ 通过直接将聚 合物沉积在预应变 PDMS 弹性衬底上，来制备一种具有 WORM 记忆特性的可拉伸忆阻器(如图 13a). 通过使用 纯共轭聚合物聚 9-乙烯咔唑(PVK) 作为活性层, 粗䊁 (a)

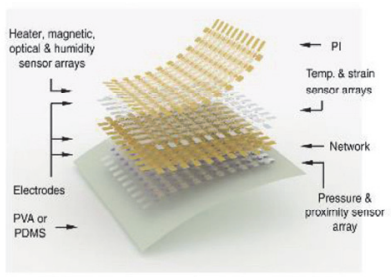

(b)

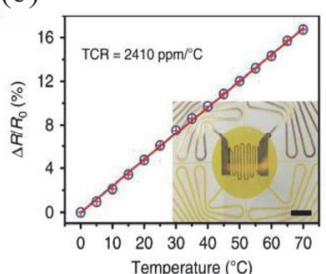

(c)

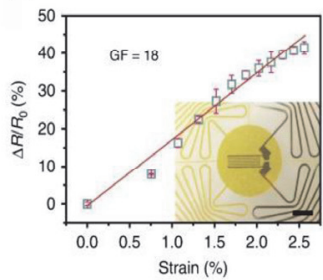

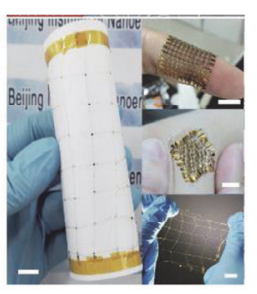
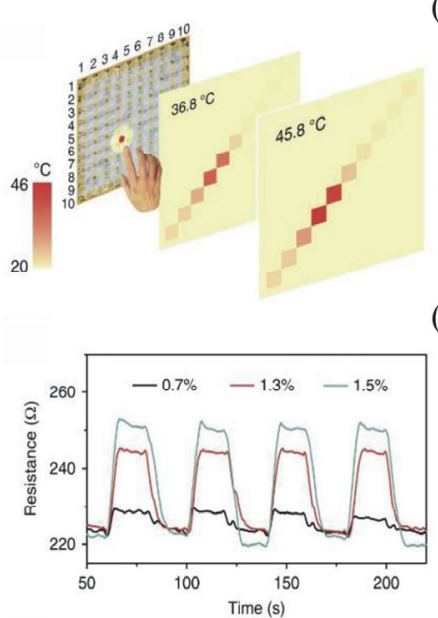

(d)
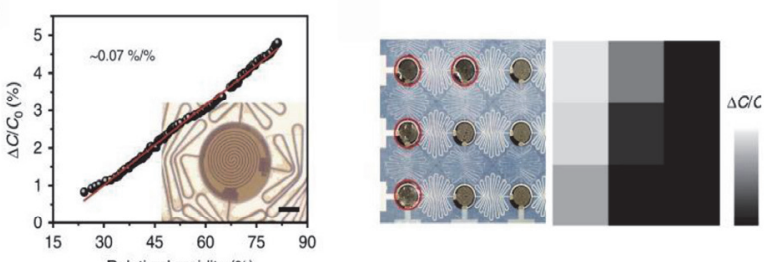

(e)
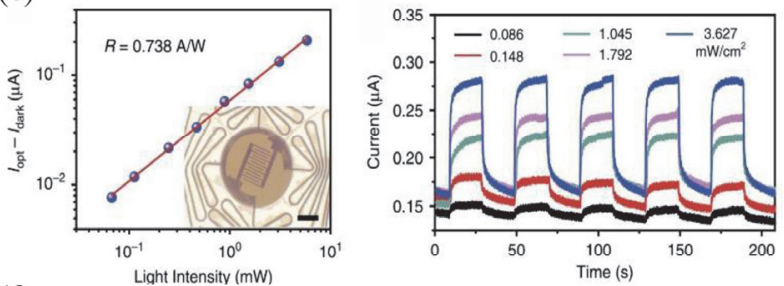

(f)
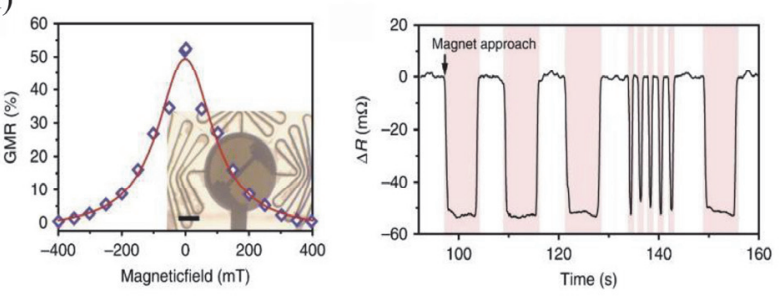

图 12 多功能可拉伸传感器

Figure 12 The multifunctional stretchable sensor

(a) Schematic illustration and photograph of the stretchable multifunctional sensor matrix networks. (b) Temperature, (c) strain, (d) humidity, (e) light, and (f) magnetic field sensing characterization of the multifunctional stretchable sensor ${ }^{[76]}$. Reprinted with permission from Ref. 76 Copyright (2018) Springer Nature. 
的 rGO 作为电极, 所制备的聚合物器件具有电阻开关特 性. 该器件还表现出典型的电双稳态行为和非易失性记 忆效应，具有高开关比 $\left(10^{4}\right)$ 、低开关电压 $(1.1 \mathrm{~V})$ 等优点 (如图 13b 13d). 此外, 该存储器显示出可靠的双稳态 电气性能，可拉伸性高达 $30 \%$, 且在 $10 \%$ 拉伸下保持时 间为 $12000 \mathrm{~s}$ 以上(如图 13e). 最重要的是, 这种方法是 通用的, 可以用于制备其他可拉伸的聚合物记忆器件.

\section{2 磁存储器}

基于材料磁性变化的磁学存储系统在传统刚性存 储器件中已发展比较成熟. 近年来, 可拉伸有机磁存储 器具有低成本、易集成等特性, 使其可能成为下一代非 易失性数据存储设备的研究热点.

光学数据存储系统是通过监测反射光束强度或偏 振变化来读取存储数据的. 将光学与磁学相结合可能是 增强数据存储可靠性的一个简便方法. Wang 等 ${ }^{[79]}$ 制备 了一种基于磁光克尔效应的可拉伸双网络水凝胶可用 于数据存储. 该水凝胶具有天然磁性, 其储存模量超过 $10^{4} \mathrm{~Pa}$, 可在 $3000 \%$ 的应变下保持完好. 水凝胶表面具 有交替的抗磁和顺磁的图案. 在磁场作用下, 水凝胶表 面会反射入射激光束并改变反射光的偏振面. 此外, 经 模拟计算知, 当条纹图案尺寸为 $5 \mu \mathrm{m}$ 时其数据容量大
约为 $1 \mathrm{MB} / \mathrm{cm}^{2}$; 若可以产生更小尺寸(如 $100 \mathrm{~nm}$ )那么 其总容量将增到 $1 \mathrm{~GB} / \mathrm{cm}^{2}$. 尽管这种磁性存储器件仍 处在概念阶段, 但其为可拉伸有机磁存储提供了一定的 参考价值.

虽然可拉伸有机磁存储器在可穿戴智能系统中有 很好的应用前景, 但是目前无论是在可拉伸有机磁性材 料的开发上, 还是在功能性器件应用上都极少有研究报 道. 据此, 有机磁存储器的发展还有很长的道路需要走.

\section{3 仿突触存储器}

仿突触存储系统受到了越来越多的关注, 它可以突 破传统的冯诺依曼计算架构, 模拟类似生物神经系统的 行为, 从而为新一代人工智能电子设备提供神经形态计 算系统. 其中忆阻器具有连续的非线性电导或电阻变化 特性, 是实现可拉伸仿突触存储器的很好选择. Liu 等 ${ }^{[80]}$ 基于高度弹性的 Ag NPs 掺杂热塑性聚氨酯制备了 一个可拉伸的突触器件, 其具有基本的突触功能包括增 强/抑制特性、长/短期可塑性、“学习-遗忘-再学习” 行 为以及依赖峰值速率和峰值振幅的可塑性. 该器件即使 在 $60 \%$ 的应变下也能很好地工作, 并可以无缝贴合在曲 面上. 同时在局部增强电场作用下, Ag NPs 团簇的运动 将形成导电长细丝, 从而可实现器件的开关. (a)

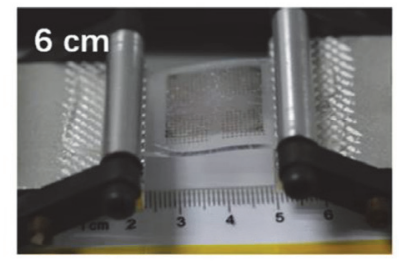

(b)

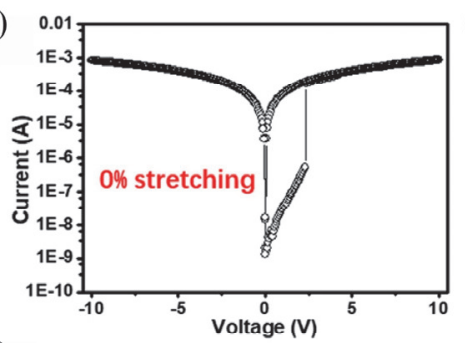

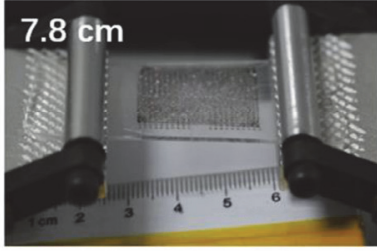

(c)

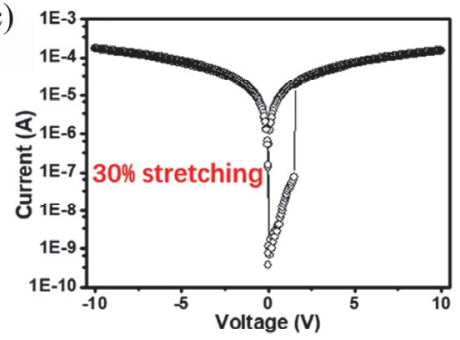

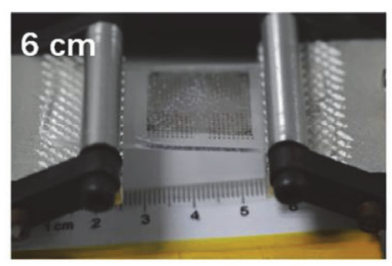

(d)

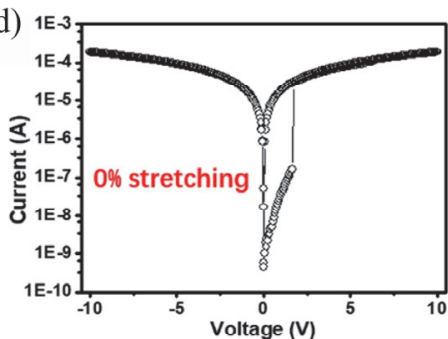

(e)

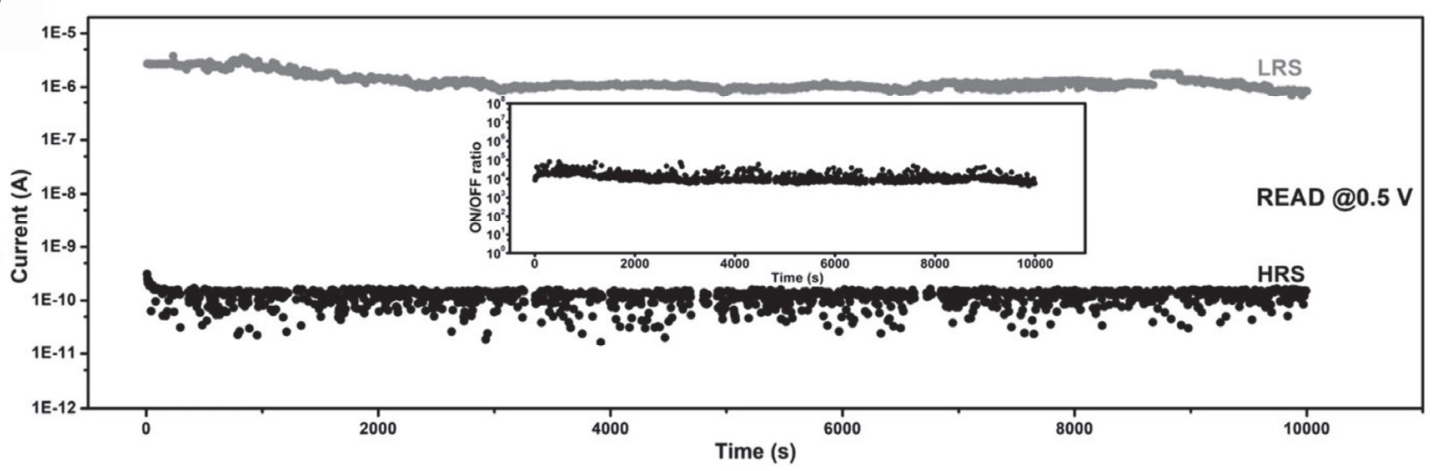

图 13 可拉伸有机忆阻器

Figure 13 The stretchable memristor

(a) Photograph of the stretchable memristor under $0 \%, 30 \%$ and freed $0 \%$ after stretching. (b $\sim \mathrm{d}) I-V$ characteristic curves in three stretching states. (e) The retention time of the stretchable memristor at $10 \%$ stretching state ${ }^{[78]}$. Reprinted with permission from Ref. 78 Copyright (2019) Springer Nature. 
尽管忆阻器一直被认为是理想的仿突触存储器件, 然而由于其随机读取、过高写入噪音和高能耗的缺点使 其难以成为低功耗突触器件的完美选择. 受大脑记忆的 启发, Salleo 等 ${ }^{[81]}$ 制备了一个电化学神经形态的有机器 件, 其与现有忆阻器具有完全不同的运行机制. 该器件 是基于对有机离子/电子导体(PEI/PEDOT:PSS) 导电性 的非易失性控制. 它本质上类似于浓度电池，在 “读” 过程中电池断开, 由于离子传导/电子阻塞电解液的作 用, 电极的电荷保持不变; 在 “写” 过程中电极中的电 荷被操纵. 因此, 该器件在开关时具有低电压和低能耗 (对于 $10^{3} \mu \mathrm{m}^{2}$ 器件能耗 $<10 \mathrm{pJ}$ ), 且在约 $1 \mathrm{~V}$ 范围内显示 出 $>500$ 个不同的非挥发性电导状态, 并可以在神经网 络仿真中实现较高的分类精度. 此外, 其被制作在灵活 的基底上, 使神经形态功能在可拉伸的电子系统中得以 整合.

到目前为止, 可拉伸有机存储器在机械性、稳定性 以及切换速度都有了很大的提高. 但是其存储性能还不 能与传统器件相妶美, 未来发展可拉伸有机存储器主要 还应集中于增大内存窗口、提高电流比、增长保留时间 和降低工作电压这四个方面 ${ }^{[82]}$, 从而使之成为高性能 存储器件应用中的首选. 高性能可拉伸有机存储器将为 可穿戴和可植入电子设备的市场普及打开大门, 并在这 一发展过程中发挥重要作用.

\section{7 其他可拉伸器件}

\section{1 致动器}

致动器在系统中主要承担着 “执行者” 的角色, 常 通过接收一种控制信号来执行控制或移动物体的行为. 可拉伸有机致动器的本征柔性促使下一代自控制电子 系统成为可能.

形状记忆聚合物膜(SMP)通常具有多稳态特性, 同 时其在较窄的温度范围内可实现机械性能大的变化 ${ }^{[83]}$, 因而被广泛使用在可拉伸有机致动器中. Zhang 等 ${ }^{[84]}$ 基 于 $\mathrm{Fe}(0)$ 粒子复合 PVDF 使用微图案法制备出了一种可 变形的 SMP 致动器, 它可用于对丙酮蒸汽多级检测. 当 该复合物膜吸附丙酮时, 可以使驱动发生变形, 而在磁 场的辅助下, 这种变形会使 Fe 粒子在 PVDF 内发生取 向, 从而实现变形到定向运动的转换. 该器件可在 $100 \%$ 的应变下, 检测高达 $160 \mathrm{mPa}$ 的应力, 表现出极好 的机械柔韧性. 此外, Shea 等 ${ }^{[83]}$ 将一组微型可拉伸加热 器安装在一层 SMP 致动器上从而集成出一种柔性皮肤. 这种致动器可以表现出大的冲程和持久的保持时间. 此 外, 该活性皮肤重量只有 $55 \mathrm{~g}$, 厚度为 $2 \mathrm{~mm}$, 同时其超 过 $99 \%$ 的分类单元是全功能性的, 可实现超过 20000 个 的循环, 因而是应用于人机界面和各种仿生电子系统的 极佳选择.

要想实现精确的动作仅仅靠简单的形状记忆是不 可靠的. 缠绕式聚合物致动器(TCAs) 具有动态检测和调
整的特点，是目前仿生(人工肌肉)效果最好的一种致动 器. Chen 等 ${ }^{[85}$ 通过将可拉伸的光学机械薄膜(SOMF)传 感器集成到 TCAs 中制备了一种具有自感知应变的致动 器(TCASA) (如图 14a). 这些传感器在 TCAs 变形过程 中有相应的颜色变化, 可以通过颜色和应变的对照来感 知应变(如图 $14 \mathrm{~b}, 14 \mathrm{c})$. 此外, 该器件具有良好的拉伸性 能, 在 200 次循环试验中表现出好的循环稳定性. 同时, 它还具有很强的自感知应变能力和应变控制能力, 因此 被视为一种极具发展前途的致动器. (a)

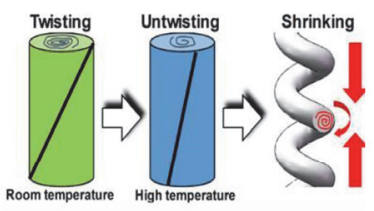

(b)

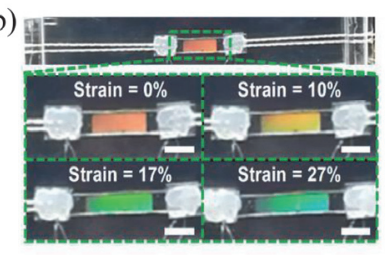

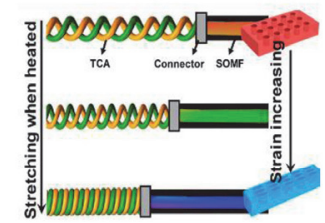

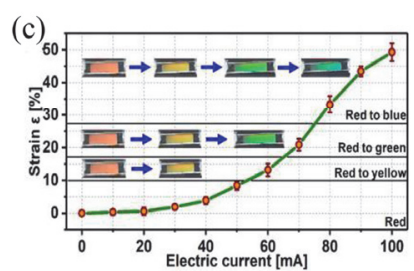

图 14 可拉伸缠绕式聚合物致动器

Figure 14 The stretchable twisted and coiled polymer actuators (a) Schematic illustration of the deformation and strain sensing mechanism of the stretchable actuator. (b) Photograph and (c) strain sensing behavior of the actuator under different electric currents ${ }^{[85]}$. Reprinted with permission from Ref. 85 Copyright (2020) American Chemical Society.

\section{2 无线通讯}

在物联网时代, 可穿戴和可植入电子产品的发展离 不开无线通讯设备. 可拉伸天线作为信号的发射和接收 装置, 可以促进移动可穿戴电子设备间的无线通信, 因 此一直受到学术界的关注.

水凝胶是生物电子学研究中的关键组件, 它具有优 异的可拉伸性、功能可调性和生物相容性, 可以将电子 器件无缝连接于生物组织上. 基于几何工程设计和微流 控技术, Kong 等 ${ }^{[86]}$ 使用液态金属制备了具有多种通道 结构的全水凝胶生物电子器件, 包括可穿戴应变传感 器、心脏贴片和近场通信(NFC) 设备, 用于无线监测人 体各种生理状况. 该类器件可以实现高达 $550 \%$ 的拉伸 应变, 其在循环拉伸、弯曲和扭转的机械作用下也能保 持稳定工作. 该设备也被证明可以在生物系统中模拟复 杂的血管网络. 此外, Messaddeq 等 ${ }^{[87]}$ 使用金属-玻璃-聚 合物纤维的天线实现了一个非侵入式非接触式呼吸传 感设备. 该天线通过与蓝牙发射器和监测站进一步集成 实现了一个无线实时监测平台. 该平台在志愿者的呼吸 能力的检测中, 可以检测到不同的呼吸模式和频率, 包 括慢、浅、不规则和快速呼吸. 以上研究表明, 可拉伸 的无线通讯设备在医疗领域以及可穿戴电子产品上有 着巨大的应用潜力. 
然而无线通讯设备常常会存在电磁干扰, 特别是基 于半导体器件的人体可穿戴设备对电磁干扰更是十分 敏感, 这就需要能够屏蔽电磁干扰(EMI)的可拉伸材料. Chen 等 ${ }^{[88}$ 制备了一种能有效减弱人体电磁吸收的可穿 戴的无线系统(如图 15a). 他们通过在乳胶上沉积由 $2 \mathrm{D}$ $\mathrm{Ti}_{3} \mathrm{C}_{2} \mathrm{~T}_{x}$ 纳米片和 SWCNTs 组成的褶皱状涂层制备了一 种超可拉伸导体, 并成功开发出了可拉伸的 EMI 屏蔽 祄垫和天线. 该设备可在松弛和拉伸状态下均表现出约 $0.01 \%$ 的恒定反射功率 $\left(\left|S_{11}\right|\right.$ 约为 $\left.-40 \mathrm{db}\right)$, 且在手臂弯曲 100 次后没有任何退化(如图 15b). 此外, 与没有任何电 磁屏蔽保护时的信号强度 $\left(\left|S_{21}\right|,-32 \mathrm{~dB}\right)$ 相比, 该设备 天线接收到的信号强度 $\left(\left|S_{21}\right|,-45 \mathrm{~dB}\right.$ 和 $\left.-52 \mathrm{~dB}\right)$ 分别 降低了 95\%和 99\%(如图 15c). 此外, 该设备可实现高达 $800 \%$ 的大面积应变, 并在循环性能测试中表现出机械 稳定的无线传输.

\section{3 集成电路}

本征可拉伸的电子电路是智能可穿戴电子产品最 终的追求目标. 可穿戴电子产品的最新研究趋势清楚地 表明，这种新兴技术将不仅局限于实验室原型，它还将 在商业产品中引起巨大的吸引力.

面对可穿戴电子对不同形式变形的需求, 目前已有 少量研究报道实现可拉伸有机电子有源阵列, 这就意味 着可拉伸有机电子器件又迈出了一大步. Bao 等 ${ }^{[89}$ 开发
出了一种基于 DPP 基聚合物的本征可拉伸晶体管阵列, 其器件密度高达 347 个 $/ \mathrm{cm}^{2}$. 这些晶体管的平均载流子 迁移率可与无定形硅相謧美，且具有极好的拉伸循环性 能. 然而受限于有机功能性材料的加工性能, 目前的可 拉伸器件仅限于单层设计 ${ }^{[90]}$. 因此进一步构建可拉伸 有机电子系统对于本征柔性电子产品的开发是至关重 要的.

$3 \mathrm{D}$ 集成可拉伸电子器件是实现大面积、高密度和 多功能整合电路的一个新方向. 目前基于无机材料的可 拉伸的三维集成技术逐渐发展起来，这对于发展可拉伸 有机电路具有相当高的参考价值. $\mathrm{Xu}$ 等 ${ }^{[2]}$ 最先基于材料 设计与微制造工程相结合制造了三维框架的可拉伸集 成电路. 他们通过把预先制备好的可拉伸电路打印到弹 性体上，并通过激光烧蚀和控制焊接构建垂直互连通 路. 该系统在垂直、水平和双轴方向分别具有 $50 \% 、 35 \%$ 和 $20 \%$ 的拉伸能力. 此外, Jacobs 等 ${ }^{[91]}$ 基于 $3 \mathrm{D}$ 构建法, 制备了一种多层交叉和垂直互连的可拉伸印刷电路板 (SPCB), 其具有高达 260\%的拉伸形变量. 同时，该方 法具有普适性, 通过集成电子和光电表面贴装器件实现 了可拉伸的有源矩阵. 该矩阵可以可逆地变形为各种 $3 \mathrm{D}$ 形状，包括但不仅限于半球形、雉形或金字塔形. 该 3D 构建法能够实现比单层方法更高的集成密度, 并可 以赋予传统单层设计难以实现的新功能. 然而, 这种方

(a)
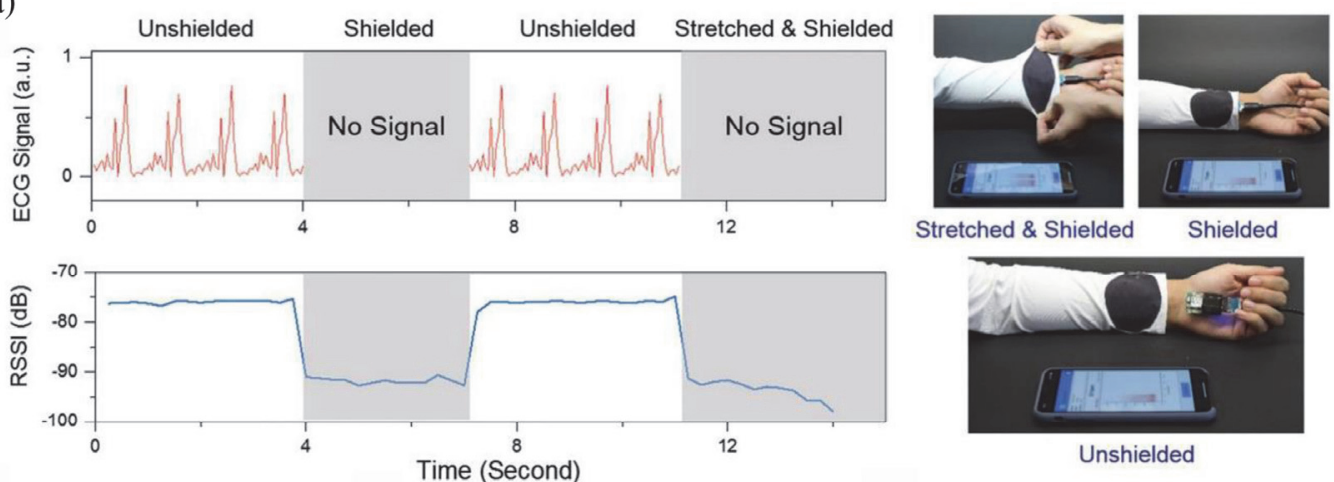

(b)

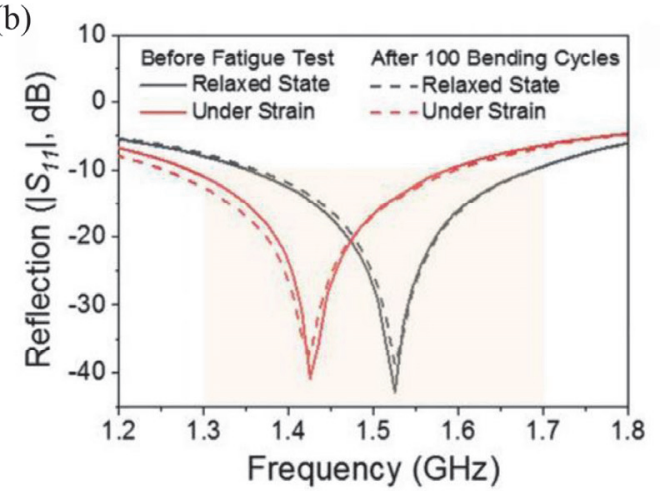

(c)

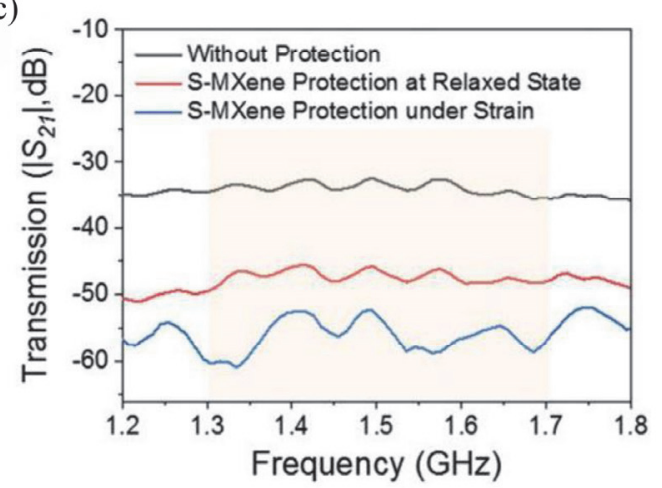

图 15 可拉伸无线通讯设备

Figure 15 The stretchable wireless communication devices

(a) Electrocardiography (ECG) and Bluetooth signals (Relative signal strength indicator, RSSI) records of the wireless system with or without shield at relaxed stretching states and the photograph at three different states. (b) Reflection $\left|S_{11}\right|$ and (c) transmission $\left|S_{21}\right|$ of the wireless system as a function of frequency with or without shield at relaxed and stretching states, respectively. ${ }^{[88]}$ Reprinted with permission from Ref. 88 Copyright (2019) WILEY-VCH. 
法仍存在着垂直互联的接口, 它是可拉伸电路系统发展 的最大阻碍. 而且这样一个复杂的几何工程导致成本增 加和整合特性降低.

\section{8 总结与展望}

在物联网时代, 新兴的可穿戴和可植入电子设备对 功能性电子器件提出了更为严苛的要求, 包括高的可拉 伸性、机械稳定性以及生物相容性等. 可拉伸有机电子 器件具有优异的机械性能、稳定的电学性能和低成本、 易集成等特点, 将为多功能电子皮肤、个性化医疗监测 和自动智能系统等领域提供更多新应用. 在过去几年 里, 无论是在晶体管、光电器件、能源存储和转换器件、 传感器、存储器等可拉伸有机器件开发上, 还是在系统 集成及功能性应用上, 可拉伸功能性电子器件都取得了 巨大的成功. 随着对可拉伸有机电子器件的深入研究, 进一步开发出高性能和多功能性电子器件才是未来器 件发展和应用的关键所在.

在本征可拉伸材料开发方面, 当前可拉伸电子器件 的活性层仍以无机材料居多, 实现其机械稳定性通常依 赖于传统的几何工程学设计, 从而导致器件的制备工艺 复杂、成本升高以及电性能和拉伸稳定性降低. 因而通 过分子结构的设计以及分子间弱相互作用的调控 ${ }^{[92]}$, 有望开发高性能的本征可拉伸有机材料, 特别是半导体 材料、光电材料、磁性材料、敏感材料以及记忆存储等 功能性材料, 将是实现本征可拉伸器件功能性应用的关 键. 此外，随着喷墨印刷、3D 打印等制造技术的发展， 材料加工性能的提高和新制备技术的开发均能推动器 件朝着大面积、高密度和多功能方向发展.

在器件性能提升方面, 当前的功能性可拉伸有机电 子器件的性能仍然无法满足实际应用和商业化要求. 通 过分子设计、形貌控制、界面改善等方法可以实现对器 件性能的有效调控. 在设计开发新型分子时应注意合理 权衡机械性能和电学性能, 同时对材料的形貌包括结晶 态、取向等进行深入的研究. 此外, 对于可拉伸功能性 器件来说, 界面的改善和新方法的引入, 如光电领域的 界面态、量子点、光磁效应等都将会推动器件向高性能 方向发展.

在多功能整合方面, 无论是不同功能器件的集成整 合还是开发多功能器件方面都面临着巨大的挑战. 一方 面, 多个不同功能器件的整合常常需要合理的电路设计 和各项性能的全面衡量. 参考 $\mathrm{Si}$ 基电路的搭建, 进一步 设计和开发本征可拉伸的有机电子系统对可拉伸电子 产业具有重要意义. 另一方面, 多功能器件要求材料本 身具备很好的兼容性以及多功能性, 巧妙地设计有机分 子和构造功能层均是开发多功能器件的有效方法. 此 外, 功能性可拉伸电子器件在电生理传感、磁存储、电 池等方面还存在着短板, 这些在未来都具有很大的发展 空间.
总之, 随着科学技术的发展, 可拉伸有机电子器件 终将在充满竞争力的电子器件中占据一席之位. 相信功 能性可拉伸电子器件的开发未来将在智能监测、仿生系 统, 甚至集成系统中发挥重要作用, 而这也正是电子器 件研究的有趣之处.

\section{作者简介}

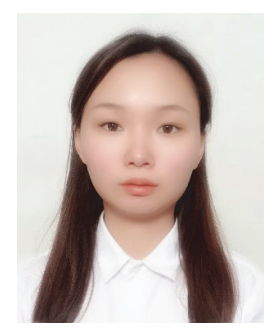

边洋爽, 中国科学院化学研究所 2019 级在读硕博生. 2019 年获北京化工大学应用化学学士学位. 研究方向为功能 性可拉伸有机电子器件.

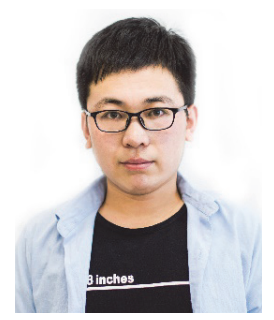

刘凯, 中国科学院化学研究所, 2018 级在读博士生. 2018 年获青岛科技大学材料学硕士学位. 研究方向为可拉伸有机 光电器件的研究.

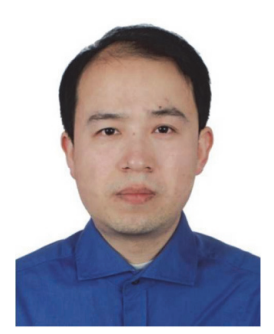

郭云龙, 男, 研究员, 博士生导师. 2010 年于中国科学院 化学研究所获博士学位, 2019 年获国家优秀青年科学基金资 助. 主要从事有机聚合物光电材料与器件以及有机-无机杂化 材料与器件的研究工作. 已在 Adv. Mater. J.Am. Chem. Soc. 、 Chem 等国际学术期刊上发表 SCI 收录论文 130 余篇. 目前为 Adv. Electron. Mater. 国际顾问委员会成员.

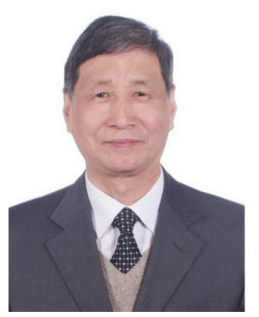

刘云圻, 男, 中国科学院院士, 第三世界科学院院士. 现 为科技部国家重点基础研究发展计划(973 计划)重大科学前沿 
领域第四届专家咨询组副组长、中国化学会理事、有机固体 专业委员会副主任. 长期从事分子材料与器件的研究, 发表 SCI 论文 700 余篇, 他人引用 4 万余次. 三次获国家自然科学 二等奖, 获北京市自然科学一等奖一项. 2014 至今, 连续入 选汤森路透全球 “高被引科学家” 目录.

\section{References}

[1] Sekitani, T.; Someya, T. Adv. Mater. 2010, 22, 2228

[2] Huang, Z. L.; Hao, Y. F.; Li, Y.; Hu, H. J.; Wang, C. H.; Nomoto, A.; Pan, T. S.; Gu, Y.; Chen, Y. M.; Zhang, T. J.; Li, W. X.; Lei, Y. S.; Kim, N.; Wang, C. F.; Zhang, L.; Ward, J. W.; Maralani, A.; Li, X. S.; Durstock, M. F.; Pisano, A.; Lin, Y.; Xu, S. Nat. Electron. 2018, $1,473$.

[3] Rogers, J. A.; Someya, T.; Huang, Y. G. Science 2010, 327, 1603.

[4] Yeo, W. H.; Kim, Y. S.; Lee, J.; Ameen, A.; Shi, L. K.; Li, M.; Wang, S. D.; Ma, R.; Jin, S. H.; Kang, Z.; Huang, Y. G.; Rogers, J. A. Adv. Mater. 2013, 25, 2773.

[5] Sekitani, T.; Noguchi, Y.; Hata, K.; Fukushima, T.; Aida, T.; Someya, T. Science 2008, 321, 1468 .

[6] Xu, S.; Zhang, Y. H.; Jia, L.; Mathewson, K. E.; Jang, K. I.; Kim, J.; Fu, H. R.; Huang, X.; Chava, P.; Wang, R. H.; Bhole, S.; Wang, L. Z.; Na, Y. J.; Guan, Y.; Flavin, M.; Han, Z. S.; Huang, Y. G.; Rogers, J. A. Science 2014, 344, 70.

[7] Yang, J. C.; Mun, J.; Kwon, S. Y.; Park, S.; Bao, Z. N.; Park, S. Adv. Mater. 2019, 31, 1904765.

[8] Oh, J. Y.; Rondeau-Gagne, S.; Chiu, Y. C.; Chortos, A.; Lissel, F.; Wang, G. J. N.; Schroeder, B. C.; Kurosawa, T.; Lopez, J.; Katsumata, T.; Xu, J.; Zhu, C. X.; Gu, X. D.; Bae, W. G.; Kim, Y.; Jin, L. H.; Chung, J. W.; Tok, J. B. H.; Bao, Z. N. Nature 2016, 539, 411.

[9] Wu, H. C.; Benight, S. J.; Chortos, A.; Lee, W. Y.; Mei, J. G.; To, J. W. F.; Lu, C. E.; He, M. Q.; Tok, J. B. H.; Chen, W. C.; Bao, Z. N. Chem. Mater. 2014, 26, 4544

[10] Wang, Y. Q.; Ding, Y.; Guo, X. L.; Yu, G. H. Nano Res. 2019, 12, 1978.

[11] Liu, K.; Guo, Y. L.; Liu, Y. Q. Sci. China-Technol. Sci. 2019, 62, 1255.

[12] Guo, Y. L.; Yu, G.; Liu, Y. Q. Adv. Mater. 2010, 22, 4427.

[13] Chortos, A.; Lim, J.; To, J. W. F.; Vosgueritchian, M.; Dusseault, T. J.; Kim, T. H.; Hwang, S.; Bao, Z. N. Adv. Mater. 2014, 26, 4253.

[14] Xu, J.; Wang, S. H.; Wang, G. J. N.; Zhu, C. X.; Luo, S. C.; Jin, L. H.; Gu, X. D.; Chen, S. C.; Feig, V. R.; To, J. W. F.; Rondeau-Gagne, S.; Park, J.; Schroeder, B. C.; Lu, C.; Oh, J. Y.; Wang, Y. M.; Kim, Y. H.; Yan, H.; Sinclair, R.; Zhou, D. S.; Xue, G.; Murmann, B.; Linder, C.; Cai, W.; Tok, J. B. H.; Chung, J. W.; Bao, Z. N. Science 2017, $355,59$.

[15] Xu, J.; Wu, H. C.; Zhu, C. X.; Ehrlich, A.; Shaw, L.; Nikolka, M.; Wang, S. H.; Molina-Lopez, F.; Gu, X. D.; Luo, S. C.; Zhou, D. S.; Kim, Y. H.; Wang, G. J. N.; Gu, K.; Feig, V. R.; Chen, S. C.; Kim, Y.; Katsumata, T.; Zheng, Y. Q.; Yan, H.; Chung, J. W.; Lopez, J.; Murmann, B.; Bao, Z. N. Nat. Materials 2019, 18, 594.

[16] Khatib, M.; Huynh, T. P.; Deng, Y. F.; Horev, Y. D.; Saliba, W.; Wu, W. W.; Haick, H. Small 2019, 15, 8.

[17] Lu, C.; Lee, W.-Y.; Gu, X.; Xu, J.; Chou, H.-H.; Yan, H.; Chiu, Y.-C.; He, M.; Matthews, J. R.; Niu, W.; Tok, J. B.-H.; Toney, M. F.; Chen, W.-C.; Bao, Z. Adv. Electron. Mater. 2017, 3, 1600311.

[18] Sang, M.; Cao, S. Z.; Lai, W. Y.; Huang, W. Acta Chim. Sinica 2015, 73,770 (in Chinese). (桑明, 曹四振, 赖文勇, 黄维, 化学学报, 2015, 73, 770.)

[19] Wang, G.-J. N.; Shaw, L.; Xu, J.; Kurosawa, T.; Schroeder, B. C.; Oh, J. Y.; Benight, S. J.; Bao, Z. Adv. Funct. Mater. 2016, 26, 7254.

[20] Mun, J.; Kang, J. H. O.; Zheng, Y.; Luo, O. O. C.; Wu, H. C.; Matsuhisa, N.; Xu, J.; Wang, G. J. N.; Yun, Y. J.; Xue, G.; Tok, J. B. H.; Bao, Z. N. Adv. Mater. 2019, 31, 1903912.

[21] Sim, K.; Rao, Z. Y.; Kim, H. J.; Thukral, A.; Shim, H.; Yu, C. J. Sci. Adv. 2019, 5, 10.

[22] Müller, C.; Goffri, S.; Breiby, D. W.; Andreasen, J. W.; Chanzy, H. D.; Janssen, R. A. J.; Nielsen, M. M.; Radano, C. P.; Sirringhaus, H.; Smith, P.; Stingelin-Stutzmann, N. Adv. Funct. Mater. 2007, 17, 2674.

[23] Peng, R.; Pang, B.; Hu, D. Q.; Chen, M. J.; Zhang, G. B.; Wang, X. H.; Lu, H. B.; Cho, K.; Qiu, L. Z. J. Mater. Chem. C 2015, 3, 3599.

[24] Mun, J.; Wang, G.-J. N.; Oh, J. Y.; Katsumata, T.; Lee, F. L.; Kang, J.; Wu, H.-C.; Lissel, F.; Rondeau-Gagne, S.; Tok, J. B. H.; Bao, Z.
Adv. Funct. Mater. 2018, 28, 1804222.

[25] Zhao, Y.; Gumyusenge, A.; He, J.; Qu, G.; McNutt, W. W.; Long, Y.; Zhang, H.; Huang, L.; Diao, Y.; Mei, J. Adv. Funct. Mater. 2018, 28, 1705584.

[26] Liang, J.; Li, L.; Tong, K.; Ren, Z.; Hu, W.; Niu, X.; Chen, Y.; Pei, Q. ACS Nano 2014, 8, 1590.

[27] Liang, J. J.; Li, L.; Chen, D.; Hajagos, T.; Ren, Z.; Chou, S. Y.; Hu, W.; Pei, Q. B. Nat. Commun. 2015, 6, 7647.

[28] Chortos, A.; Koleilat, G. I.; Pfattner, R.; Kong, D. S.; Lin, P.; Nur, R.; Lei, T.; Wang, H. L.; Liu, N.; Lai, Y. C.; Kim, M. G.; Chung, J. W.; Lee, S.; Bao, Z. N. Adv. Mater. 2016, 28, 4441.

[29] Li, L.; Liang, J. J.; Gao, H. E.; Li, Y.; Niu, X. F.; Zhu, X. D.; Xiong, Y.; Pei, Q. B. ACS Appl. Mater. Interfaces 2017, 9, 40523.

[30] Savagatrup, S.; Makaram, A. S.; Burke, D. J.; Lipomi, D. J. $A d v$ Funct. Mater. 2014, 24, 1169.

[31] Yu, Z. B.; Niu, X. F.; Liu, Z. T.; Pei, Q. B. Adv. Mater. 2011, 23, 3989.

[32] Liang, J.; Li, L.; Niu, X.; Yu, Z.; Pei, Q. Nat. Photonics 2013, 7, 817.

[33] Wu, X.; Lan, S.; Hu, D.; Chen, Q.; Li, E.; Yan, Y.; Chen, H.; Guo, T. J. Mater. Chem. C 2019, 7, 9229.

[34] Zhong, J.; Wu, X.; Lan, S.; Fang, Y.; Chen, H.; Guo, T. ACS Photonics 2018, 5, 3712.

[35] Yang, H.; Liu, Y.; Wu, X.; Yan, Y.; Wang, X.; Lan, S.; Zhang, G.; Chen, H.; Guo, T. Adv. Electron. Mater. 2019, 1900864.

[36] Kang, M.; Lee, S. A.; Jang, S.; Hwang, S.; Lee, S. K.; Bae, S.; Hong, J. M.; Lee, S. H.; Jeong, K. U.; Lim, J. A.; Kim, T. W. ACS Appl. Mater. Interfaces 2019, 11, 22575.

[37] Han, S. T.; Zhou, Y.; Roy, V. A. L. Adv. Mater. 2013, 25, 5425.

[38] Hong, S. Y.; Kim, M. S.; Park, H.; Jin, S. W.; Jeong, Y. R.; Kim, J. W.; Lee, Y. H.; Sun, L.; Zi, G.; Ha, J. S. Adv. Funct. Mater. 2019, 29, 9.

[39] Zhu, C. X.; Chortos, A.; Wang, Y.; Pfattner, R.; Lei, T.; Hinckley, A. C.; Pochorovski, I.; Yan, X. Z.; To, J. W. F.; Oh, J. Y.; Tok, J. B. H.; Bao, Z. N.; Murmann, B. Nat. Electron. 2018, 1, 183.

[40] Zhu, C.; Wu, H. C.; Nyikayaramba, G.; Bao, Z. N.; Murmann, B. IEEE Electron Device Lett. 2019, 40, 1630.

[41] Zang, Y.; Zhang, F.; Huang, D.; Di, C.-a.; Zhu, D. Adv. Mater. 2015 $27,7979$.

[42] Shim, H.; Sim, K.; Ershad, F.; Yang, P. Y.; Thukral, A.; Rao, Z.; Kim, H. J.; Liu, Y. H.; Wang, X.; Gu, G. Y.; Gao, L.; Wang, X. R.; Chai, Y.; Yu, C. J. Sci. Adv. 2019, 5, 11.

[43] Molina-Lopez, F.; Gao, T. Z.; Kraft, U.; Zhu, C.; Ohlund, T.; Pfattner, R.; Feig, V. R.; Kim, Y.; Wang, S.; Yun, Y.; Bao, Z. Nat. Commun. 2019, 10, 2676.

[44] Matsuhisa, N.; Jiang, Y.; Liu, Z. Y.; Chen, G.; Wan, C. J.; Kim, Y.; Kang, J.; Tran, H.; Wu, H. C.; You, I.; Bao, Z. N.; Chen, X. D. Adv. Electron. Mater. 2019, 5, 1900347.

[45] Li, Y. Z.; Wang, N. X.; Yang, A. N.; Ling, H. F.; Yan, F. Adv. Electron. Mater. 2019, 5,7

[46] Yin, D.; Feng, J.; Ma, R.; Liu, Y. F.; Zhang, Y. L.; Zhang, X. L.; Bi, Y. G.; Chen, Q. D.; Sun, H. B. Nat. Commun. 2016, 7, 11573.

[47] Kim, T. H.; Lee, C. S.; Kim, S.; Hur, J.; Lee, S.; Shin, K. W.; Yoon, Y. Z.; Choi, M. K.; Yang, J.; Kim, D. H.; Hyeon, T.; Park, S.; Hwang, S. ACS Nano 2017, 11, 5992.

[48] Hu, D.; Xu, X.; Miao, J.; Gidron, O.; Meng, H. Materials 2018, 11, 184.

[49] Wang, X.; Sun, J.; Dong, L.; Lv, C.; Zhang, K.; Shang, Y.; Yang, T.; Wang, J.; Shan, C.-X. Nano Energy 2019, 58, 410.

[50] Shin, H.; Sharma, B. K.; Lee, S. W.; Lee, J.-B.; Choi, M.; Hu, L.; Park, C.; Choi, J. H.; Kim, T. W.; Ahn, J.-H. ACS Appl. Mater. Interfaces 2019, 11, 14222.

[51] Wang, J. X.; Lee, P. S. Nanophotonics 2017, 6, 435.

[52] Larson, C.; Peele, B.; Li, S.; Robinson, S.; Totaro, M.; Beccai, L.; Mazzolai, B.; Shepherd, R. Science 2016, 351, 1071.

[53] Tan, Y. J.; Godaba, H.; Chen, G.; Tan, S. T. M.; Wan, G.; Li, G.; Lee, P. M.; Cai, Y.; Li, S.; Shepherd, R. F.; Ho, J. S.; Tee, B. C. K. Nat. Materials 2020, 19, 182.

[54] Chou, H. H.; Nguyen, A.; Chortos, A.; To, J. W. F.; Lu, C.; Mei, J. G.; Kurosawa, T.; Bae, W. G.; Tok, J. B. H.; Bao, Z. N. Nat. Commun. 2015, 6, 8011.

[55] Yin, D.; Jiang, N.-R.; Liu, Y.-F.; Zhang, X.-L.; Li, A.-W.; Feng, J.; Sun, H.-B. Light-Sci. Appl. 2018, 7, 262.

[56] An, T. C.; Ling, Y. Z.; Gong, S.; Zhu, B. W.; Zhao, Y. M.; Dong, D. S.; Yap, L. W.; Wang, Y.; Cheng, W. L. Adv. Mater. Technol. 2019, 4, 1800473 . 
[57] Huang, Y.; Zhong, M.; Huang, Y.; Zhu, M. S.; Pei, Z. X.; Wang, Z. F.; Xue, Q.; Xie, X. M.; Zhi, C. Y. Nat. Commun. 2015, 6, 10310.

[58] Park, S.; Lee, H.; Kim, Y. J.; Lee, P. S. NPG Asia Mater. 2018, 10, 11.

[59] Siddiqui, S.; Lee, H. B.; Kim, D.-I.; Le Thai, D.; Hanif, A.; Lee, N.-E. Adv. Energy Mater. 2019, 9, 1701520.

[60] Pu, X.; Liu, M. M.; Chen, X. Y.; Sun, J. M.; Du, C. H.; Zhang, Y.; Zhai, J. Y.; Hu, W. G.; Wang, Z. L. Sci. Adv. 2017, 3, 1700015.

[61] Zou, Y.; Tan, P.; Shi, B.; Ouyang, H.; Jiang, D.; Liu, Z.; Li, H.; Yu, M.; Wang, C.; Qu, X.; Zhao, L.; Fan, Y.; Wang, Z. L.; Li, Z. Nat. Commun. 2019, 10, 2695.

[62] Zhao, S.; Zhu, R. Acta Chim. Sinica 2019, 77, 1250 (in Chinese). (赵帅, 朱荣, 化学学报, 2019, 77, 1250.)

[63] Qian, X.; Su, M.; Li, F. Y.; Song, Y. L. Acta Chim. Sinica 2016, 74, 565 (in Chinese). (钱金金, 苏萌, 李风煜, 宋延林, 化学学报, 2016, 74, 565.)

[64] Jian, M. Q.; Xia, K. L.; Wang, Q.; Yin, Z.; Wang, H. M.; Wang, C. Y.; Xie, H. H.; Zhang, M. C.; Zhang, Y. Y. Adv. Funct. Mater. 2017, 27, 1606066

[65] Liao, X. Q.; Wang, W. S.; Wang, L.; Tang, K.; Zheng, Y. J. ACS Appl. Mater. Interfaces 2019, 11, 2431.

[66] Chen, H. T.; Su, Z. M.; Song, Y.; Cheng, X. L.; Chen, X. X.; Meng, B.; Song, Z. J.; Chen, D. M.; Zhang, H. X. Adv. Funct. Mater. 2017, 27, 1604434.

[67] Boutry, C. M.; Kaizawa, Y.; Schroeder, B. C.; Chortos, A.; Legrand, A.; Wang, Z.; Chang, J.; Fox, P.; Bao, Z. N. Nat. Electron. 2018, 1, 314.

[68] Cataldi, P.; Dussoni, S.; Ceseracciu, L.; Maggiali, M.; Natale, L.; Metta, G.; Athanassiou, A.; Bayer, I. S. Adv. Sci. 2018, 5, 10.

[69] Wang, X. D.; Zhang, Y. F.; Zhang, X. J.; Huo, Z. H.; Li, X. Y.; Que, M. L.; Peng, Z. C.; Wang, H.; Pan, C. F. Adv. Mater. 2018, 30, 8.

[70] Ren, Z. W.; Nie, J. H.; Xu, L.; Jiang, T.; Chen, B. D.; Chen, X. Y.; Wang, Z. L. Adv. Funct. Mater. 2018, 28, 9.

[71] Trung, T. Q.; Dang, T. M. L.; Ramasundaram, S.; Toi, P. T.; Park, S. Y.; Lee, N. E. ACS Appl. Mater. Interfaces 2019, 11, 2317.

[72] Trung, T. Q.; Ramasundaram, S.; Hwang, B. U.; Lee, N. E. $A d v$. Mater. 2016, 28, 502.

[73] Song, Z. L.; Huang, Z.; Liu, J. Y.; Hu, Z. X.; Zhang, J. B.; Zhang, G. Z.; Yi, F.; Jiang, S. L.; Lian, J. B.; Yan, J.; Zang, J. F.; Liu, H. ACS Sens. 2018, 3, 1048.

[74] Park, J.; Kim, J.; Kim, S. Y.; Cheong, W. H.; Jang, J.; Park, Y. G.; Na, K.; Kim, Y. T.; Heo, J. H.; Lee, C. Y.; Lee, J. H.; Bien, F.; Park, J. U. Sci. Adv. 2018, 4, 9841.
[75] Wang, Z.; Wang, X.; Li, M.; Gao, Y.; Hu, Z.; Nan, T.; Liang, X.; Chen, H.; Yang, J.; Cash, S.; Sun, N.-X. Adv. Mater. 2016, 28, 9370.

[76] Hua, Q. L.; Sun, J. L.; Liu, H. T.; Bao, R. R.; Yu, R. M.; Zhai, J. Y.; Pan, C. F.; Wang, Z. L. Nat. Commun. 2018, 9, 244.

[77] Hsu, L. C.; Shih, C. C.; Hsieh, H. C.; Chiang, Y. C.; Wu, P. H.; Chueh, C. C.; Chen, W. C. Polym. Chem. 2018, 9, 5145.

[78] Ban, C. Y.; Wang, X. J.; Zhou, Z.; Mao, H. W.; Cheng, S.; Zhang, Z. P.; Liu, Z. D.; Li, H.; Liu, J. Q.; Huang, W. Sci. Rep. 2019, 9, 7.

[79] Gui, Q. Y.; Zhou, Y.; Liao, S. L.; He, Y. L.; Tang, Y. F.; Wang, Y. P. Soft Matter 2019, 15, 393.

[80] Yang, M. H.; Zhao, X. L.; Tang, Q. X.; Cui, N.; Wang, Z. Q.; Tong, Y. H.; Liu, Y. C. Nanoscale 2018, 10, 18135.

[81] van de Burgt, Y.; Lubberman, E.; Fuller, E. J.; Keene, S. T.; Faria, G. C.; Agarwal, S.; Marinella, M. J.; Talin, A. A.; Salleo, A. Nat. Materials 2017, 16, 414.

[82] Zhou, L.; Mao, J. Y.; Ren, Y.; Han, S. T.; Roy, V. A. L.; Zhou, Y. Small 2018, 14, 1703126.

[83] Besse, N.; Rosset, S.; Zarate, J. J.; Shea, H. Adv. Mater. Technol. 2017, 2, 1700102.

[84] Wei, J.; Wang, F.; Zhang, L. ACS Appl. Mater. Interfaces 2018, 10, 29161.

[85] Zhao, P.; Xu, B.; Zhang, Y.; Li, B.; Chen, H. ACS Appl. Mater. Interfaces 2020, 12, 15716.

[86] Liu, Y. R. N.; Yang, T. Y.; Zhang, Y. Y.; Qu, G.; Wei, S. S.; Liu, Z.; Kong, T. T. Adv. Mater. 2019, 31, 1902783.

[87] Roudjane, M.; Bellemare-Rousseau, S.; Khalil, M.; Gorgutsa, S.; Miled, A.; Messaddeq, Y. Sensors 2018, 18, 973.

[88] Li, Y.; Tian, X.; Gao, S.-P.; Jing, L.; Li, K.; Yang, H.; Fu, F.; Lee, J. Y.; Guo, Y.-X.; Ho, J. S.; Chen, P.-Y. Adv. Funct. Mater. 2020, 30, 1907451.

[89] Wang, S. H.; Xu, J.; Wang, W. C.; Wang, G. J. N.; Rastak, R.; Molina-Lopez, F.; Chung, J. W.; Niu, S. M.; Feig, V. R.; Lopez, J.; Lei, T.; Kwon, S. K.; Kim, Y.; Foudeh, A. M.; Ehrlich, A.; Gasperini, A.; Yun, Y.; Murmann, B.; Tok, J. B. H.; Bao, Z. N. Nature 2018, 555, 83.

[90] Oh, J. Y.; Bao, Z. N. Adv. Sci. 2019, 6, 1900186.

[91] Biswas, S.; Schoeberl, A.; Hao, Y. F.; Reiprich, J.; Stauden, T.; Pezoldt, J.; Jacobs, H. O. Nat. Commun. 2019, $10,8$.

[92] Zhang, S. X.; Shao, X. F. Acta Chim. Sinica 2018, 76, 531 (in Chinese). (张尚秃, 邵向锋, 化学学报, 2018, 76, 531.)

(Cheng, B.) 